\title{
Postnatal development and maturation of layer 1 in the lateral prefrontal cortex and its disruption in autism
}

\author{
Iris Margalit Trutzer ${ }^{1,2}$, Miguel Ángel García-Cabezas ${ }^{3}$ and Basilis Zikopoulos ${ }^{1,2^{*}}$ (D)
}

\begin{abstract}
Autism is a neurodevelopmental connectivity disorder characterized by cortical network disorganization and imbalance in excitation/inhibition. However, little is known about the development of autism pathology and the disruption of laminar-specific excitatory and inhibitory cortical circuits. To begin to address these issues, we examined layer 1 of the lateral prefrontal cortex (LPFC), an area with prolonged development and maturation that is affected in autism. We focused on layer 1 because it contains a distinctive, diverse population of interneurons and glia, receives input from feedback and neuromodulatory pathways, and plays a critical role in the development, maturation, and function of the cortex. We used unbiased quantitative methods at high resolution to study the morphology, neurochemistry, distribution, and density of neurons and myelinated axons in post-mortem brain tissue from children and adults with and without autism. We cross-validated our findings through comparisons with neighboring anterior cingulate cortices and optimally-fixed non-human primate tissue. In neurotypical controls we found an increase in the density of myelinated axons from childhood to adulthood. Neuron density overall declined with age, paralleled by decreased density of inhibitory interneurons labeled by calretinin (CR), calbindin (CB), and parvalbumin (PV). Importantly, we found PV neurons in layer 1 of typically developing children, previously detected only perinatally. In autism there was disorganization of cortical networks within layer 1: children with autism had increased variability in the trajectories and thickness of myelinated axons in layer 1, while adults with autism had a reduction in the relative proportion of thin axons. Neurotypical postnatal changes in layer 1 of LPFC likely underlie refinement of cortical activity during maturation of cortical networks involved in cognition. Our findings suggest that disruption of the maturation of feedback pathways, rather than interneurons in layer 1, has a key role in the development of imbalance between excitation and inhibition in autism.
\end{abstract}

Keywords: Autism neuropathology, Laminar architecture, Postnatal axon myelination, Feedback pathways, Inhibitory neuron, Anterior cingulate cortex

\section{Introduction}

Autism is a neurodevelopmental disorder that is characterized by changes in neural communication that affect diverse sensory-motor processes such as attention and social interaction $[2,36,66]$. Changes in frontal networks, including increased short-range and decreased long-range communication as well as changes in synchronization between cortical areas during tasks,

\footnotetext{
* Correspondence: zikopoul@bu.edu

${ }^{1}$ Human Systems Neuroscience Laboratory, Boston University, 635 Commonwealth Ave., Room 401D, Boston, MA 02215, USA

2Program in Neuroscience, Boston University, Boston, MA 02215, USA

Full list of author information is available at the end of the article
}

have been described in individuals with autism [12, 24, $31,61,63,110,124]$. Anatomical studies have identified changes in the distribution and density of neurons belonging to multiple subtypes within frontal cortices $[1$, 52, 130] and myelinated axons below the frontal lobes in autism $[129,130,133]$ that likely underlie these findings. However, little is known about the development of cortical pathology and the disruption of laminar-specific excitatory pathways and inhibitory circuits in the affected frontal cortical networks.

The development of cortical network pathology in the lateral prefrontal cortex (LPFC) is of particular interest because LPFC is involved in attention and the cognitive 
processes that are affected in autism and undergoes prolonged postnatal development and maturation [13, 23, $52,71,115,116,129-131]$. Layer 1 plays a significant role in the prenatal patterning of the cortex and postnatally is a chief recipient of feedback and neuromodulatory pathways in LPFC, making it an ideal candidate for the study of the development of laminar-specific pathway pathology in autism.

Layer 1 contains a distinctive set of morphologically diverse local circuit neurons along with varied populations of astrocytes, oligodendrocytes, and microglia $[11,40,81,97$, $102,125,127]$. Feedback connections from cortical areas as well as the thalamus, amygdala, and neuromodulatory systems target layer $1[5,7,10,15,48,60,87,112,128]$, where they interact with local excitatory and inhibitory circuits and affect spatiotemporal characteristics of cortical activity patterns $[17,26,33]$. In prenatal development, the intrinsic Cajal-Retzius cells of layer 1 secrete reelin to direct the development of the distinct cortical layers [39, 53, 93]. Studies of the development of layer 1 have examined mostly the pre- and postnatal maturation of Cajal-Retzius neurons and few other cell types [78, 82, 88, 90, 102, 114, 126, 127]. However, we know little about the postnatal changes in the diverse cellular populations of layer 1 and their relationship with the maturation of the pathways that terminate there, which serve to transition this layer from a developmental mediator to a processor of feedback input. Importantly, changes in the expression of factors that determine the maturation and activity of cortical networks have been described in layer 1 neurons in LPFC in neurodevelopmental connectivity disorders such as schizophrenia [104] and autism [115]. The effects of these disruptions on the cellular organization and axonal networks within layer 1 in childhood and adulthood are unknown.

To begin to address these issues we used post-mortem brain tissue from individuals with and without autism (ages 3-67 years) to systematically and quantitatively examine the postnatal development of excitatory and inhibitory circuits in layer 1 of the human LPFC. We cross-validated our findings through comparisons with neighboring anterior cingulate cortices (ACC) of neurotypical individuals and optimally-fixed non-human primate tissue. We provide evidence for significant changes in the density of neurons and maturation of pathways in typical postnatal development from childhood through late adulthood. We notably found that PV-immunoreactive neurons, which have previously only been shown in layer 1 of neonates, persist in layer 1 through late adolescence, likely influencing the signaling dynamics of the inhibitory neurons in layer 1. Comparison of the structure of layer 1 in typically developing individuals with that of individuals with autism revealed significant disruption in the developmental trajectory and structure of pathways, but not neurons, in children and adults with autism.
Pathology included increased variability in axon orientation and changes in the size of myelinated axons. These findings suggest that persistent disruption of feedback pathways in LPFC in children and adults may underlie excitatory-inhibitory imbalance, cortical network disorganization, and atypical focusing and shifting of attention in autism.

\section{Methods}

\section{Experimental design}

The aim of this study was to examine the structural, neurochemical, and molecular characteristics of layer 1 of the human LPFC in children and adults with and without a diagnosis of autism, a disorder in which imbalance of excitation-inhibition has been shown using multiple approaches $[27,49,103]$. First, we examined the postnatal structural and neurochemical maturation of layer 1 in neurotypically developing individuals to define the typical developmental trajectory. We compared typical development with the trends seen in children and adults with autism in order to probe whether there were patterns of cortical disorganization that could underlie autism symptomatology.

We focused our analysis specifically on layer 1 of LPFC because it is a major target of feedback pathways and is involved in the performance of high-order organizational tasks and attentional regulation, which are affected in autism. We systematically and quantitatively examined neurons and myelinated axons in layer 1 of LPFC and separately analyzed the excitatory and inhibitory components of the local neural network (Fig. 1). To describe the typical range of fundamental anatomical and neurochemical features of layer 1 , we qualitatively studied and compared layer 1 of neurotypical subjects in the LPFC and in the ACC, two regions of the prefrontal cortex with different laminar structures [131]. We used archival processed tissue from ACC and LPFC of rhesus macaques as an important control to validate the results from the analysis of human tissue, and placed our findings within the context of classical and modern studies of the cellular composition, circuitry, and connectivity of layer 1 . Tissue was labeled with Nissl and Gallyas stain to identify the overall structural characteristics of layer 1, and we used specific antibodies to label glia, inhibitory neurons, and markers of cortical stability and plasticity. Within this framework of analyses we were able to describe dynamic changes in network structure during neurotypical development, and we were able to identify pathological changes in patterns of cortical organization underlying information processing during development in autism.

\section{Post-mortem tissue acquisition and processing}

Post-mortem age-matched tissue from 32 individuals (16 control, 16 autism) was acquired from the Harvard Brain 

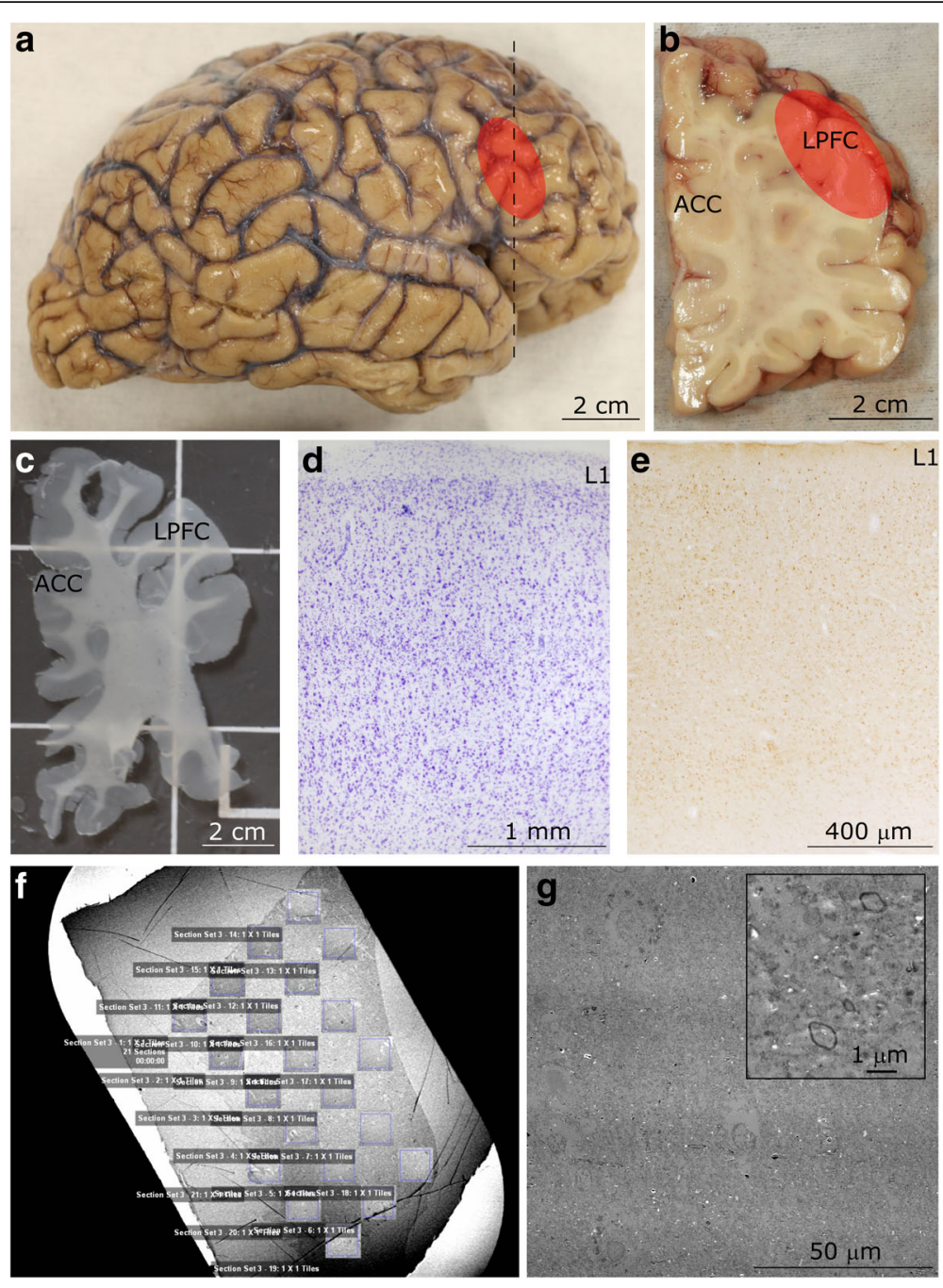

Fig. 1 Experimental design. a Lateral view of the right hemisphere of the adult human brain. The region of the lateral prefrontal cortex (LPFC) analyzed in this study is shown in red. $\mathbf{b}$ Coronal tissue slab taken from the frontal cortex at the level marked by the dotted line in $\mathbf{a}$. The red overlay highlights the LPFC at the level of Brodmann's area 46. c Representative free-floating tissue section, cut at $50 \mu \mathrm{m}$, used for staining. d-e Representative cortical columnar regions of interest processed for Nissl $\mathbf{d}$ and calretinin $\mathbf{e}$ in the LPFC. Layer 1 (L1) is labeled in both columns. $\mathbf{f}$ Representative image of a $50 \mathrm{~nm}$-thick section from LPFC gray matter on a pioloform-coated slot grid to show the unbiased systematic sampling scheme used to analyze axons at the EM level. This scheme also resembles the sampling used in quantitative analysis of cell and axon populations at the light microscope. $\mathbf{g}$ Representative high-resolution electron micrograph of an ultrathin section (50 $\mathrm{nm}$ ), sampled from $\mathbf{f}$, and acquired using a scanning-transmission electron microscope (STEM) system. Myelinated axon profiles can be identified by the darkly stained, electron dense ring of myelin surrounding the axolemma (shown at high magnification in inset). This representative image was taken from layer 5 of LPFC

Tissue Resource Center, the Autism Tissue Program, the Institute for Basic Research in Developmental Disabilities, the University of Maryland Brain and Tissue Bank, and the National Disease Research Interchange (NDRI). Autism diagnosis was assessed through the Autism Diagnostic Interview-Revised (ADI-R) and many cases had corresponding Autism Diagnostic Observation Schedule (ADOS) scores. Four subjects had comorbid diagnoses of seizure disorder (HSB4640, AN 08792), depression (AN 18892) and schizophrenia (AN 06746); findings in these cases, which have also been successfully used in previous studies [133], fell within the ranges for each group. All available cases were included in the analyses for this study. Case AN18892 (B-4871) was used solely in a qualitative assessment of ACC. All other cases were included in quantitative immunohistochemistry analyses. Cases 4021, 4029, AN 03221, 5144, 1182, B-5173, B-6232, B-6677, 451, 4203, 4337, 3835, B-6004, B-5353, B-4981, HAW, and HAY were used for electron microscopy analyses. Clinical characteristics and other data on human subjects can be found in Table 1. 
Cases were matched based on availability, and brains were well preserved and had low post-mortem interval (PMI) (average PMI < 24h). To ensure appropriate age-matching, HAW and HAY, which are older control cases, were excluded from the comparison with individuals with autism but were included in the analysis of neurotypical development and aging [average age of individuals with autism: Children $=6.9 \pm 3.6$ years $(1 \mathrm{~F})$, Adults $=36.0 \pm 7.1$ years $(1 \mathrm{~F})$; average age of agematched neurotypical individuals: Children $=9.5 \pm 3.2$ years $(2 \mathrm{~F})$, Adults $=40.0 \pm 4.2$ years $(2 \mathrm{~F})]$. Tissue was fixed in $10 \%$ formalin, cryoprotected in $30 \%$ sucrose, and stored at $-20^{\circ} \mathrm{C}$ in antifreeze solution $(30 \%$ ethylene glycol, 30\% glycerol, 40\% $0.05 \mathrm{M} \mathrm{PB}$ with $0.05 \%$ azide).

We evaluated layer 1 in granular LPFC and dysgranular ACC in order to identify the fundamental composition of layer 1, which we assessed by noting both common organizational patterns and distinct features of areas that otherwise have gross differences in laminar organization. In adult cases, coronal blocks of tissue corresponding to area 46 in the LPFC or area 32 in the ACC were identified using atlases as guides and were excised for sectioning $[6,77,100,101,122,123]$. In children, coronally-cut slabs of the frontal lobe were divided into dorsal and ventral blocks and sectioned for analysis.

Table 1 Cases and Clinical Characteristics

\begin{tabular}{|c|c|c|c|c|c|}
\hline Subject Number & Age (years) & Condition & Sex & PMI (h) & Cause of Death \\
\hline AN03345 (B-6399) & 3 & $\mathrm{AU}$ & M & 4 & Cardiac arrest \\
\hline 4021 & 3.3 & $\mathrm{AU}$ & M & 15 & Accidental drowning \\
\hline 4029 & 3.8 & $\mathrm{AU}$ & M & 13 & Accidental drowning \\
\hline 5308 & 4.5 & $A U$ & M & 21 & Accident, injuries \\
\hline AN08873 (B-5569) & 5 & $A U$ & M & 25.5 & Accidental drowning \\
\hline AN03221 & 7 & $\mathrm{AU}$ & M & 11 & Accidental drowning \\
\hline 5144 & 7.2 & $A U$ & M & 3 & Cancer \\
\hline HSB4640 & 8.5 & $\mathrm{AU}$ & M & 14 & Asthma attack, seizure \\
\hline AN01293 (B-6349) & 9 & $A U$ & M & 4 & Cardiopulmonary arrest \\
\hline 1182 & 10 & $\mathrm{AU}$ & $\mathrm{F}$ & 24 & Smoke inhalation \\
\hline AN04682 (B-7079) & 15 & $\mathrm{AU}$ & M & 23.2 & Asphyxia, hanging \\
\hline AN-08792 (B-5173) & 30 & $\mathrm{AU}$ & M & 20 & Gastro-intestinal bleeding \\
\hline AN-11989 (B-6677) & 30 & $\mathrm{AU}$ & M & 16 & Congestive heart failure \\
\hline AN-18892 (B-4871) & 31 & $\mathrm{AU}$ & M & 99 & Shooting \\
\hline AN-07770 (B-6232) & 40 & $\mathrm{AU}$ & $\mathrm{F}$ & 33 & Respiratory arrest \\
\hline AN-06746 (B-4541) & 44 & $\mathrm{AU}$ & M & 31 & Acute myocardial infarction \\
\hline 451 & 4.6 & CTR & M & 15 & Accidental drowning \\
\hline 4203 & 7.8 & CTR & M & 24 & Respiratory insufficiency \\
\hline 4337 & 8.2 & CTR & M & 16 & Blunt force neck injury \\
\hline 1706 & 8.6 & $C T R$ & $\mathrm{~F}$ & 20 & Rejection of cardiac transplant \\
\hline 3835 & 9.6 & CTR & $\mathrm{F}$ & 8 & Asphyxia \\
\hline 1548 & 10 & CTR & M & 0 & Unknown \\
\hline 1670 & 14 & CTR & M & 5 & Respiratory distress \\
\hline 4722 & 14 & CTR & M & 16 & Multiple traumatic injuries \\
\hline B-4786 & 36 & CTR & M & 20 & Myocardial infarction \\
\hline B-6004 & 36 & CTR & $\mathrm{F}$ & 18 & Unknown \\
\hline $\mathrm{HCD}$ & 38 & CTR & M & 18.9 & Cardiac arrest \\
\hline B-5353 & 41 & CTR & $\mathrm{F}$ & 14 & Unknown \\
\hline B-4981 & 42 & CTR & M & 18 & Myocardial infarction \\
\hline $\mathrm{HCF}$ & 47 & CTR & M & 21.9 & Cardiac arrest \\
\hline HAW & 58 & CTR & $\mathrm{F}$ & 30 & Pancreatic cancer \\
\hline HAY & 67 & CTR & M & 30 & Pancreatic cancer \\
\hline
\end{tabular}

PMI: Post-mortem Interval 
Sections from equivalent levels from all cases were matched to control for structural differences along the rostro-caudal axis of our region of interest (ROI). Tissue blocks were frozen in $-70^{\circ} \mathrm{C}$ isopentane to prepare for sectioning into 10 series of $50 \mu \mathrm{m}$-thick sections using a cryostat (CM 1500, Leica) or freezing microtome (American Optical). Few sections from cases B-6232 and B-6677 were also cut at $20 \mu \mathrm{m}$ and stained for Nissl analysis. Results from these thin sections did not differ from those obtained from thicker sections. Alternatively, additional blocks from adult cases that were not frozen were rinsed in $0.1 \mathrm{M} \mathrm{PB}$ and cut in series of $50 \mu \mathrm{m}$-thick sections using vibratomes (series 1000, Pelco; Precisionary VF-700, Precisionary Instruments Inc.). One series of sections was mounted immediately following sectioning on chrome-alum gelatin-coated slides in order to preserve the series. These sections were used as a reference to determine the position of floating sections on the rostro-caudal axis.

We evaluated layer 1 in LPFC and ACC in optimally-fixed brain tissue from non-human primates (Macaca mulatta) as an important control in order to assess the effects of PMI and quality of fixation on our quantitative analysis and to further cross-validate our findings. We obtained archival tissue from young adult rhesus monkeys (aged 2-4 years) that were used for experiments and cared for in accordance with the requirements of the Institutional Animal Care and Use Committee (IACUC) of Boston University. Monkey cases RAJ (F), RAN, RAT (2 years old, F), RAV, RAW (F), RAX, RAY ( 3 years old, F), RBB ( 2 years old, F), RBI ( 2.8 years old, F), RBN ( 2 years old, $\mathrm{M}$ ), RBO (3 years old, $\mathrm{M}$ ), RBS (3.5 years old, F), RBT ( 4 years old, F) and RBU ( 4 years old, M) were used for analyses. Monkeys were perfused with $4 \%$ paraformaldehyde (RAJ, RAN, RAT, RAV, RAW, RBB) or $4 \%$ paraformaldehyde with $0.2 \%$ glutaraldehyde (RAX, RAY, RBI, RBN, RBO, RBS, RBT, RBU) as previously described $[46,131]$. The brains of these animals were then cryoprotected in graded sucrose solutions and frozen in $-70{ }^{\circ} \mathrm{C}$ isopentane for rapid, uniform freezing. Brains were sectioned into $40 \mu \mathrm{m}$-thick sections (RAJ, RAN, RAT, RAV, RAW, RAX, RAY) or $50 \mu$ m-thick sections (RBB, RBI, RBN, RBO, RBS, RBT, $R B U$ ) in 10 matched series, with one series mounted immediately for reference, as described above [46].

\section{Histology and immunohistochemistry for light microscopy}

Nissl-stained sections were used to analyze overall neuron density in layer 1 . Sections were stained as previously described [46]. Briefly, sections underwent de-fatting in a 1:1 solution of chloroform and $100 \%$ ethanol for $2-3 \mathrm{~h}$ prior to rehydration using a gradient of alcohols and $\mathrm{dH}_{2} \mathrm{O}$. Sections were stained using $0.05 \%$ thionin blue for $15 \mathrm{~min}$. Stained tissue was dehydrated and differentiated in graded alcohols and xylenes prior to coverslipping using Entellan (Merck).

Gallyas myelin labeling of human tissue followed published protocols $[43,132]$. Briefly, sections were rinsed in distilled water and then incubated in pyridine (2/3; P3681 Fischer Scientific) with glacial acetic acid (1/3; ARK2183 Sigma-Aldrich) for $30 \mathrm{~min}$ at room temperature. Sections were washed again in distilled water followed by incubation in the impregnation solution $[0.1 \mathrm{~g}$ ammonium nitrate (A7455 Sigma-Aldrich) and $0.1 \mathrm{~g}$ silver nitrate (S181-25 Fischer Scientific) per $100 \mathrm{ml}$ of distilled water, $\mathrm{pH}$ 7.5] for a minimum of $30 \mathrm{~min}$ at room temperature in the dark. Sections were rinsed in $0.5 \%$ acetic acid (A6283 Sigma-Aldrich) and were then incubated in the developing solution [Three components: A, $25 \mathrm{~g}$ sodium carbonate (S-263 Fischer Scientific) in $500 \mathrm{ml}$ distilled water; B, $1 \mathrm{~g}$ ammonium nitrate (A7455 Sigma-Aldrich), $1 \mathrm{~g}$ silver nitrate (S181-25 Fischer Scientific) and $5 \mathrm{~g}$ silico-tungstic acid (383,341 Sigma-Aldrich) in $500 \mathrm{ml}$ distilled water; C, $75 \mathrm{ml}$ of solution $\mathrm{B}$ and $1.75 \mathrm{ml}$ of $4 \%$ paraformaldehyde (O4042 Fischer Scientific) in $500 \mathrm{ml}$ distilled water] under microscopic control until the proper level of stain was achieved. $150 \mathrm{ml}$ of solution A was combined with $75 \mathrm{ml}$ of solution B and $75 \mathrm{ml}$ of solution $\mathrm{C}$, in that order, to create the impregnation solution. After developing, sections were washed in 1\% acetic acid (A6283 Sigma-Aldrich) and then in distilled water before incubation in 5\% sodium thiosulfate (S-1648 Sigma-Aldrich) to stabilize the reaction. Sections were finally washed in distilled water and mounted and coverslipped as described above.

Inhibitory interneurons and glial cells in human tissue were labeled through immunohistochemistry as previously reported [32, 47, 84, 130]. Three functionally distinct classes of interneurons are distinguished in the primate cortex through their differential expression of calcium-binding proteins. Calbindin (CB)-expressing neurons target the distal dendrites of pyramidal neurons, while calretinin (CR)-expressing interneurons target $\mathrm{CB}$ neurons [28]. Parvalbumin (PV)-expressing interneurons synapse on the perisomal regions of their targets and are therefore strongly inhibitory [28]. We labeled glial cells with antibodies against CD44 (to label interlaminar astrocytes), excitatory amino acid transporter-2 (EAAT2, to label astrocytes), and ionized calcium-binding adaptor molecule 1 (Iba-1, to label microglia). Information regarding the antibodies used in this study is included in Table 2.

In short, sections were rinsed in $0.01 \mathrm{M} \mathrm{PBS,} \mathrm{pH} \mathrm{7.4,}$ followed by $10 \%$ serum matching the species of the secondary antibody, $5 \%$ bovine serum albumin, and $0.1 \%$ Triton X-100 in $0.01 \mathrm{M}$ PBS blocking solution for $1 \mathrm{~h}$ and incubated for 2 days at $4{ }^{\circ} \mathrm{C}$ in primary antibody. Some sections were double-labeled, while others were single-labeled. The sections were rinsed in PBS and 
Table 2 Properties of the Antibodies Used

\begin{tabular}{llllll}
\hline Antigen & Animal Raised & Source & Catalog Number & Research Resource Identifiers (RRID) & Dilution \\
\hline Calbindin & Mouse & Swant & 300 & AB_10000347 & AB_2721225 \\
Calbindin & Rabbit & Swant & CB38 & AB_10000320 \\
Calretinin & Mouse & Swant & $6 B 3$ & AB_2313763 \\
Calretinin & Rabbit & Swant & $7699 / 4$ & $1: 2000$ \\
a-CamKIl & Mouse & Boehringer Mannheim & $1,481,703$ & AB_2314079 \\
CD44 & Rat & Calbiochem & 217,594 & AB_211582 & $1: 2000$ \\
GABA & Rabbit & ImmunoStar (Diasorin Inc.) & 20,094 & AB_572234 \\
GAD67 & Mouse & Millipore (Chemicon) & MAB5406 & AB_2278725 \\
GFAP & Rabbit & Sigma & G-9269 & AB_477035 \\
EAAT2 & Mouse & BD Biosciences & 611,654 & AB_399172 \\
Iba-1 & Goat & Abcam & ab5076 & AB_2224402 \\
NeuN & Mouse & Millipore (Chemicon) & MAB377 & AB_2298772 \\
Parvalbumin & Mouse & Swant & PV235 & AB_10000343 \\
Parvalbumin & Mouse & Sigma & P3088 & AB_477329 \\
goat anti-mouse IgG, Biotinylated & Goat & Vector Laboratories & BA-9200 & AB_2336171 \\
goat anti-rabbit IgG, Biotinylated & Goat & Vector Laboratories & BA-1000 & AB_2313606 \\
goat anti-rat IgG, Biotinylated & Goat & Vector Laboratories & BA-9400 & AB_2336202 \\
horse anti-goat IgG, Biotinylated & Horse & Vector Laboratories & BA-9500 & AB_2336123 \\
horse anti-mouse IgG, Biotinylated & Horse & Vector Laboratories & BA-2000 & AB_2313581 & $1: 250$ \\
\hline
\end{tabular}

incubated overnight at $4{ }^{\circ} \mathrm{C}$ with biotinylated goat anti-mouse and/or goat anti-rabbit and/or goat anti-rat secondary antibodies and thoroughly rinsed with PBS. An avidin-biotin-peroxidase kit was used to label CB-, $\mathrm{PV}-$, or CR-expressing neurons with diaminobenzidine (Zymed Laboratories) for single-label experiments and an SG peroxidase kit was used to label cells with the immunohistochemistry chromogen SG (Vector Laboratories) in double-labeling experiments. Glial cells were labeled using avidin-biotin-peroxidase kit with diaminobenzidine (Zymed Laboratories). Control experiments with the omission of primary and/or secondary antibodies showed no nonspecific labeling, as previously reported $[46,47,130]$. Sections were mounted and coverslipped as described above. All sections were examined to ensure consistent, sufficient labeling for accurate quantification. Sections from cases were omitted from individual quantitative analyses if staining was inconsistent and caused concern about the accuracy of quantitative results.

We used archival processed non-human primate tissue (Macaca mulatta) to examine additional components of layer 1 in the primate cortex and to validate the results of the analysis of human LPFC. Tissue was labeled with Nissl and Gallyas stain, as described above. Sections were labeled through immunohistochemistry with antibodies against NeuN (an alternate method for labeling neurons, which specifically targets neuronal nuclei), gamma-Aminobutyric acid (GABA), glutamate decarboxylase (GAD67), PV, CB, CR, alpha subunit of calmodulin kinase II ( $\alpha$-CamKII), Iba- 1 , and glial fibrillary acidic protein (GFAP). All staining protocols have been previously described $[46,47,130]$, and were similar to the processing of human tissue (above).

\section{Processing for electron microscopy}

Tissue was processed for EM using a high-contrast method [133]. Sections processed for EM were adjacent to Nissl- and immunohistochemistry-labeled sections. Sections were washed in $0.1 \% \mathrm{~PB}$ and postfixed in $6 \%$ glutaraldehyde using a variable-wattage microwave to improve tissue penetration of fixative and other reagents. Sections were first rinsed in $0.1 \mathrm{M}$ cacodylate buffer followed by $0.1 \%$ tannic acid prior to serial rinses in heavy metal solutions (1\% osmium tetroxide with $1.5 \%$ potassium ferrocyanide, $0.1 \mathrm{~g}$ of thiocarbohydrazide, and finally $2 \%$ osmium tetroxide). Heavy metals impregnate lipid bilayers, creating contrast between membranes and other tissue components. Sections were washed with water, stained overnight in $1 \%$ uranyl acetate, and were finally stained with lead aspartate prior to dehydration in serial alcohols. Dehydrated tissue was cleared in propylene oxide and embedded in LX112 resin which was hardened between sheets of Aclar film at $60^{\circ} \mathrm{C}$ for long-term storage. 
We identified cortical gray matter in processed tissue using a dissecting microscope. ROIs containing gray matter were cut from the Aclar sheets and reembedded in LX112 resin blocks for sectioning at the ultramicrotome. $50 \mathrm{~nm}$-thick sections were cut and collected on single-slot pioloform grids for imaging with a scanning electron microscope (SEM). $1 \mu \mathrm{m}$-thick (semi-thin) sections were cut and mounted on gelatin-coated slides and stained with toluidine blue powder in distilled water. Semi-thin sections were dried and then covered with toluidine blue solution for $1 \mathrm{~min}$ on a hot plate prior to being rinsed with water and coverslipped as described $[129,131,133]$.

\section{Imaging and quantitative analysis Light microscopy}

Layer 1 was identified in stained sections using reference maps $[6,77,100,101,123,124]$. Neurons were identified and quantified in Nissl-stained sections using a validated algorithm [46]. We quantified neuron density in multiple ROIs on one series of coronal sections per case. Sequential sections in children were on average $500 \mu \mathrm{m}$ apart, while those in adults were on average $400 \mu \mathrm{m}$ apart. We used an unbiased statistical sampling method to sample the areas of interest using commercially available software (StereoInvestigator, MicroBrightfield). The counting frame for these analyses was set to $150-180 \mu \mathrm{m}$ with a height of $8 \mu \mathrm{m}$ and grid spacing of $150-560 \mu \mathrm{m}$. The thickness of the section was measured at each counting site and a guard zone was set at the top of each section $(2 \mu \mathrm{m})$. We calculated the density of neurons in layer 1 (neuron density, also referred to as the packing density) by dividing the estimated number of cells in layer 1 in each case by the measured volume of layer $1 \mathrm{in} \mathrm{mm}^{3}$. Quantification of immunolabeled tissue was performed at 400x, while Nissl-stained tissue was quantified at 1000x. Quantification of immunohistochemistry-labeled tissue sections was exhaustive, meaning that all counting frames were analyzed (counting grid and frame size were equal).

We measured the area fraction of neurons in highresolution images of Nissl-stained tissue (thionin and toluidine blue) and the area fraction of axons in toluidine blue- and osmium-stained sections. Area fraction is a measure of the percent of the overall tissue surface area which contains axons or neurons. We used a consistent protocol to modify brightness and contrast of each image through adjustment of the levels of the histogram to maximize the black and white tonal range, while keeping the midtones unchanged (value 1.00). We thresholded images to increase the contrast between neurons, myelinated axons, and neuropil and to ensure reproducibility. Thresholding was performed using a standardized function within ImageJ that maintains the image moment, or weighted average of the intensity of the image pixels, in the whole image: this separates the image into meaningful gray-level classes. All background correction occurred automatically at image acquisition, and was maintained through the use of identical levels of luminescence for all image acquisitions. Standardized functions within ImageJ performed all measurements.

To place our findings within the context of postnatal maturation and myelination of frontal cortical layer 1 in the human brain we studied myelination patterns throughout the neurotypical lifespan using the classical atlas of cortical myelination by Kaes [62]. Kaes processed tissue using Weigart's myelin stain (Max Wolter's variant) in thinly cut tissue (under $50 \mu \mathrm{m}$ ) and examined 12 regions of the cortex from both hemispheres across 45 human brains, ranging from 3 months to 97 years old. His study focused on the thickness of the layers, defined by their myelin level, during postnatal development [92]. Comparison of the density of myelin in adults in Kaes' material and our processed sections allowed us to match analyzed ROIs between datasets based on their similarity. Among Kaes' ROIs, the anterior and posterior frontal lobes included the anterior and lateral prefrontal cortices, while Kaes' Gyrus Fornicatus ROI is the cingulate cortex, including the ACC, in line with Kaes' descriptions [62] and previous assessments [92].

We scanned the illustrations of columns from 31 brains ( 3 months -65 years old, mean age $=27$ years old; 7 female) from the atlas (original publication) at high resolution and used the scans to estimate the plot profiles of acquired cortical columns in ImageJ. Kaes' published atlas used in this study is out of copyright and the book is in the public domain. We estimated the average gray level (optical density) of layer 1 in these columns using the acquired plot profile.

\section{Electron microscopy}

We acquired high-resolution images using a scanning electron microscope (Zeiss Gemini 300 with STEM detector, Atlas 5 software) at magnifications ranging from 2000x to 50,000x as previously described [133].

Maps of processed tissue labeled with toluidine blue were used to identify layer 1 in EM-processed tissue. We sampled grids in an unbiased way, similar to that described above and as shown in Fig. 1. We acquired all images at $30 \mathrm{~nm}$ resolution and performed all analyses of these images in Image J. We directly circled all myelinated axon profiles and obtained the measurement of the angle, major and minor diameter, surface area, and perimeter of each axon.

Axon segment trajectory measurements were acquired directly from the outlined profiles of axons analyzed in individual electron micrographs. For each outlined axon profile we estimated the major and minor diameter, 
aspect ratio, and the angle trajectory of the major diameter. Previous studies have successfully used this method to describe changes in axon trajectory in autism [133], and these measurements have been shown to correlate with the local trajectory of myelinated axons using 3D-reconstruction [129]. We processed the raw measurements of the axon segment angles following a previously reported protocol [133]. Briefly, we aligned the peak angle for each section to 90 degrees to account for bias from image acquisition, and we calculated the standard deviation of the angles for each case. We used the proportion of elongated axons (axons with aspect ratio $>3$ ) as a weighting factor to produce the final reported standard deviation measurement. Axons with aspect ratio $<3$ appeared more circular, and the angles of these axons were not a reliable indicator of the axon trajectory. Axons with aspect ratios $>3$ were more elongated, and produced reliable measurements.

\section{Statistics and cross-validation}

All values are reported as the mean \pm standard deviation (SD). To ensure that the results of these studies had statistical power, we selected a sampling fraction and studied a tissue volume that resulted in an error of under $10 \%$, as described $[44,47,129,131,133]$. Data was statistically analyzed using ANCOVAs in SPSS (IBM) in order to determine correlations with age, cause of death, PMI, gender, and other identifying characteristics of our sample. The Levene statistical test, which assesses the homogeneity of variances between studied groups, was used to assess differences in variability between groups. Results were considered statistically significant with a $p$-value under 0.05 .

Many independent experimental and analytical methods, ranging from light and electron microscopy, toluidine blue labeling, and myelin staining were used to cross-validate the results and minimize the effects of any potential experimental bias. We used archival monkey tissue to further validate our findings in human tissue, ensuring that factors in human tissue analysis and acquisition, such as PMI or quality of fixation, did not bias our conclusions. Additionally, multiple researchers analyzed subsets of the same data.

\section{Results}

We studied excitatory and inhibitory elements in LPFC layer 1 in neurotypically developing children and adults with the aim of identifying age-related changes in the structure of layer 1 . We compared normative changes with those seen in individuals with a diagnosis of autism in order to identify atypical age-related changes in network structure that may influence the development of autism symptomatology. To validate our findings in human tissue, we qualitatively examined layer 1 in the eulaminate LPFC and in the limbic-dysgranular ACC in human and non-human primate tissue to assess key characteristics of this layer that underlie its dynamic role in cortical development and function.

\section{Myelinated axons in layer 1: Normative development and changes in autism}

We analyzed the structure and organization of myelinated axons in neurotypical individuals and compared normative developmental trends with the trends seen in individuals with autism (Fig. 2). In layer 1, myelinated axons derive from cortical and subcortical excitatory feedback pathways and local axons from excitatory and inhibitory neurons. Images of axons in representative children and adults with and without a diagnosis of autism are shown in Fig. 3. Analysis of neurotypical children and adults is shown in the left panels and a comparison with individuals with autism is in the right panels.

\section{Normative development of myelinated axons in layer 1}

We examined functionally relevant subpopulations of myelinated axons in layer 1 in neurotypically developing children and adults and assessed the increase in myelination using two complementary approaches. First, we measured the surface area occupied by myelinated axons using area fraction measures in osmicated and Nissl toluidine blue-stained sections. Myelinated axons occupied little of the surface area of layer 1 in childhood (mean \pm SD: $0.89 \pm 0.29 \%$ ); this increased significantly $(p=0.000)$ to $5.4 \pm 0.44 \%$ in adults (Fig. 2a). We also calculated the angle trajectories of all myelinated axon profiles, and we evaluated the organization of the network within layer 1 by determining the variability in the trajectories of the axons in all cases. Variability in myelinated axon trajectory deviation was small in childhood, ranging from $3.6^{\circ}$ $5.0^{\circ}\left(4.20 \pm 0.56^{\circ}\right)$, and increased in adulthood $(6.08 \pm$ $\left.2.60^{\circ}\right)$, potentially reflecting the increased myelination of more diverse pathways with age (Fig. 2b).

Analysis of the laminar density of myelin in layer 1 of the anterior and posterior prefrontal cortex and the cingulate cortex (gyrus fornicatus) from cases used in Kaes' atlas was in line with our quantitative analysis at the light and electron microscope. We observed a gradual and significant increase in myelination of layer 1 with age: anterior and posterior frontal regions had a steeper rate of increase in myelination during development than the cingulate cortex and higher levels of myelin in adulthood $[\mathrm{p}$ (Frontal Lobes $)=0.005 ; \mathrm{p}$ (Gyrus Fornicatus) $=0.041]$ (Fig. 2d).

Axon caliber is correlated both with the length of the pathway and with efficiency of signal transduction: thick axons participate mainly in long-range connections and transmit signals faster than thin axons, which are mainly 


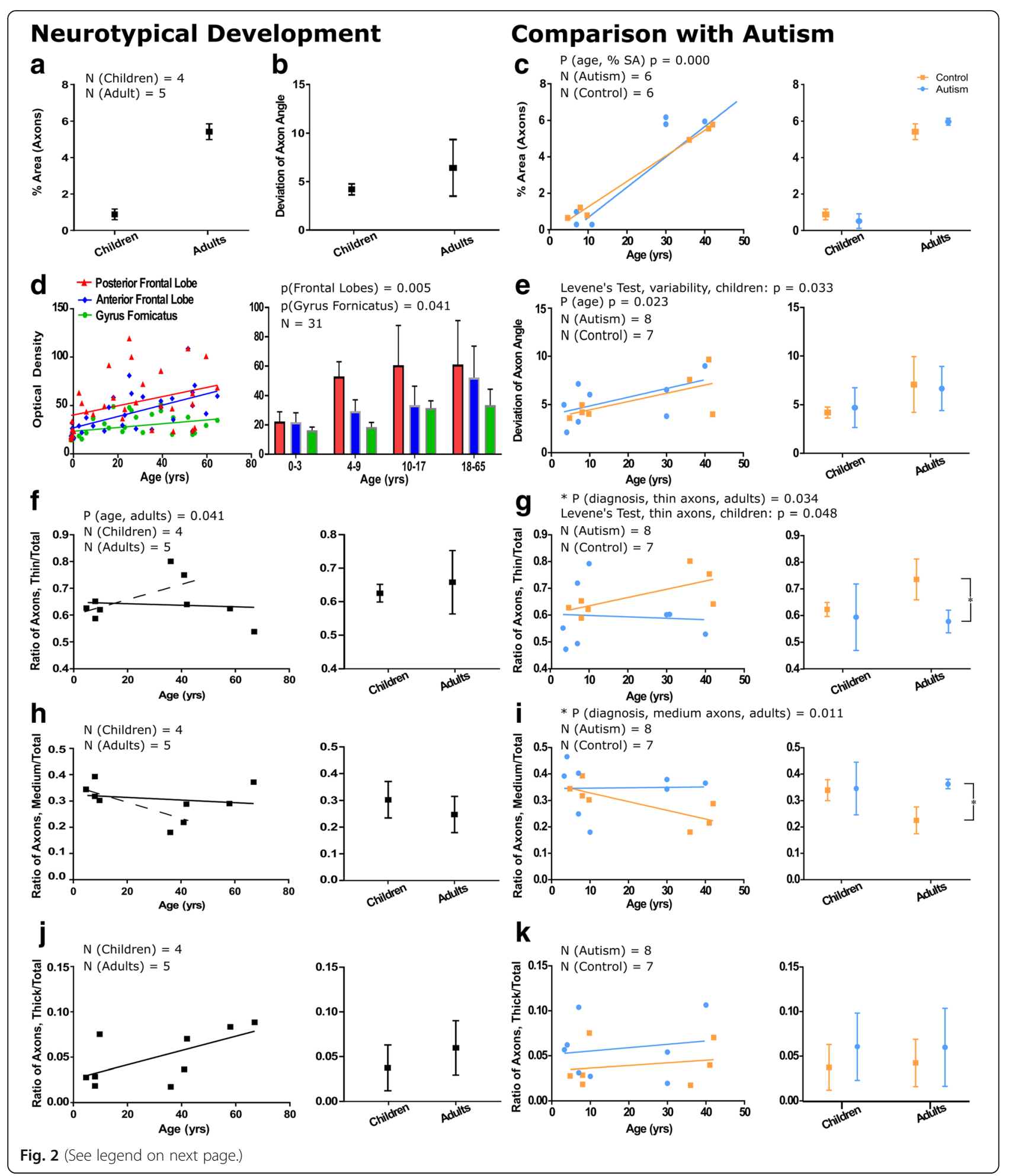


(See figure on previous page.)

Fig. 2 Myelinated axons in layer 1 of prefrontal cortex show significant changes in development and in autism. a The percent surface area occupied by axons in children is low $(0.89 \pm 0.29 \%)$, but increases significantly to $5.4 \pm 0.44 \%$ in adults. b Axon trajectories are less variable in children than in adults in neurotypical development, suggesting increased myelination of diverse pathways in adulthood. c The percent surface area occupied by myelinated axons was similar in autism and neurotypical groups. The percent surface area occupied by axons increased significantly in adults from both groups $(p=0.000$ ), shown on a case-by-case basis (left graph) and average by age group (right graph). $\mathbf{d}$ We analyzed images of layer 1 from the atlas of Kaes [62] to cross-validate our findings. Trends of myelin development in layer 1 of the anterior and posterior frontal lobes, which are similar to anterior and lateral prefrontal cortices, and Gyrus Fornicatus, which includes the anterior cingulate cortex, showed increases in myelination with age. In the posterior frontal lobe, layer 1 in children aged 0-3 was less myelinated than older children and adults. The anterior frontal lobe and anterior cingulate cortex had a more prolonged period of myelination and overall had a lower level of myelination compared with the posterior frontal lobe. e In children with autism there was a significant increase in the variability of axon trajectory heterogeneity when compared to neurotypical children $(p=0.033)$. In adults, this variability was similar between groups. $\mathbf{f}$ The relative proportion of thin axons remained approximately stable through neurotypical development. The solid line shows the overall stable trend, the dotted line shows the increase in the relative proportion of thin axons when comparing children and young adults. There was a trend towards a decline in the proportion of thin axons with age in adulthood. $\mathbf{g}$ The proportion of thin axons in neurotypical children was on average $64 \%$; in children with autism, there was significant variation in the percentage of axons that were thin (range: $47-75 \%$ ). Neurotypical adults had a higher average proportion of thin axons than adults with autism (mean control $=73.6 \pm 7.7 \%$, mean autism $=57.8 \pm 4.1 \%, p=0.034$ ). $\mathbf{h}$ The relative proportion of medium-caliber axons remained approximately stable through neurotypical development. The solid line shows the overall stable trend, the dotted line shows the decrease in the relative proportion of medium-caliber axons when comparing children and young adults. There was a trend towards an increase in the proportion of medium-caliber axons with age in adulthood. $\mathbf{i}$ There was significant variability in the proportion of medium-caliber axons in children with autism. In adulthood, there was a significant increase in the relative proportion of mediumcaliber axons in adults with autism compared with neurotypical adults (mean control $=22.5 \pm 5.1 \%$, mean autism $=36.2 \pm 1.8 \%, p=0.011$ ). $\mathbf{j}$ There was a trend towards an increase in the relative proportion of thick axons in neurotypical development. $\mathbf{k}$ Developmental trends in the proportion of thick axons were not different between autism and control groups. Left panels in e-k present developmental trends on a case-by-case basis, and right panels show mean \pm SD for children and adults with and without an autism diagnosis. Autism cases 4021, 4029, AN 03221, 5144, 1182, B-5173, B-6232, B-6677, and control cases 451, 4203, 4337, 3835, B-6004, B-5353, and B-4981 were used for electron microscopy comparisons. Control adults HAW and HAY were included in the study of neurotypical development and aging

found in short-range connections [58, 131]. Therefore, we assessed the sizes of myelinated axons in layer 1 in order to identify potential changes in the relative proportion of different pathways and overall changes in the efficiency of signaling at different developmental stages. We divided axons into three categories: thin axons (diameter $<0.84 \mu \mathrm{m})$, medium-caliber axons $(0.84 \mu \mathrm{m}<$ diameter $<1.18 \mu \mathrm{m}$ ) and thick axons (diameter $>$ $1.18 \mu \mathrm{m})$, as described [129, 131, 133]. In neurotypically developing children, $62.3 \pm 2.6 \%$ of myelinated axons in layer 1 were thin while $33.9 \pm 3.9 \%$ were of medium caliber; few thick myelinated axons were present (Fig. 2f, h, j). There were no significant differences in these proportions between children and adults. In adults there was an age-related decline in the relative proportion of thin myelinated axons and increase in the relative proportion of medium-caliber myelinated axons in layer $1(p=0.041)$, potentially relating to changes in pruning or branching characteristics in the neuropil that may occur with normal aging.

\section{Changes in the myelinated axons of layer 1 in autism}

We found significant changes in the organization and structure of myelinated axons in layer 1 of both children and adults with autism when compared with neurotypical individuals. In order to ensure appropriate agematching of subjects, we excluded unmatched older control adults from comparisons.
The density of myelinated axons increased with age in individuals with autism and controls (Fig. 2a, c) ( $p=0.000$; no difference between autism and control, $p=0.349$ ). The variability in myelinated axon trajectory deviation was significantly larger in children with autism compared to neurotypical children $(p=0.033)$ : this difference did not persist in adults, although there was a significant increase in myelinated axon trajectory deviation with age $(p=0.023)$ (Fig. 2e). This finding suggests that there is significant local circuit disorganization in individuals with autism beginning early in postnatal development. In children with autism, we found significantly increased variability in the proportion of myelinated thin axons (SD Autism = $12.5 \%$, SD Control $=2.6 \%, p=0.048$ ) (Fig. 2g). In adults with autism, the proportion of myelinated thin axons in layer 1 was significantly lower than that in controls (mean Control $=73.6 \pm 7.7 \%$, mean Autism $=57.9 \pm 4.1 \%, p=0.034$ ) (Fig. 2g), accompanied by a significant increase in the proportion of myelinated medium-caliber axons (mean Control $=22.5 \pm 5.1 \%$, mean Autism $=36.2 \pm 1.8 \%, \quad p=0.011$ ) (Fig. 2i). These changes were not associated with parallel alterations in the density of axons in adults with autism $(p=0.390)$.

\section{Neuron population in layer 1: Normative development and comparison with autism}

Next, we examined the overall neuronal population of layer 1 along with three functionally distinct subclasses of neurons labeled by the calcium-binding proteins PV, 

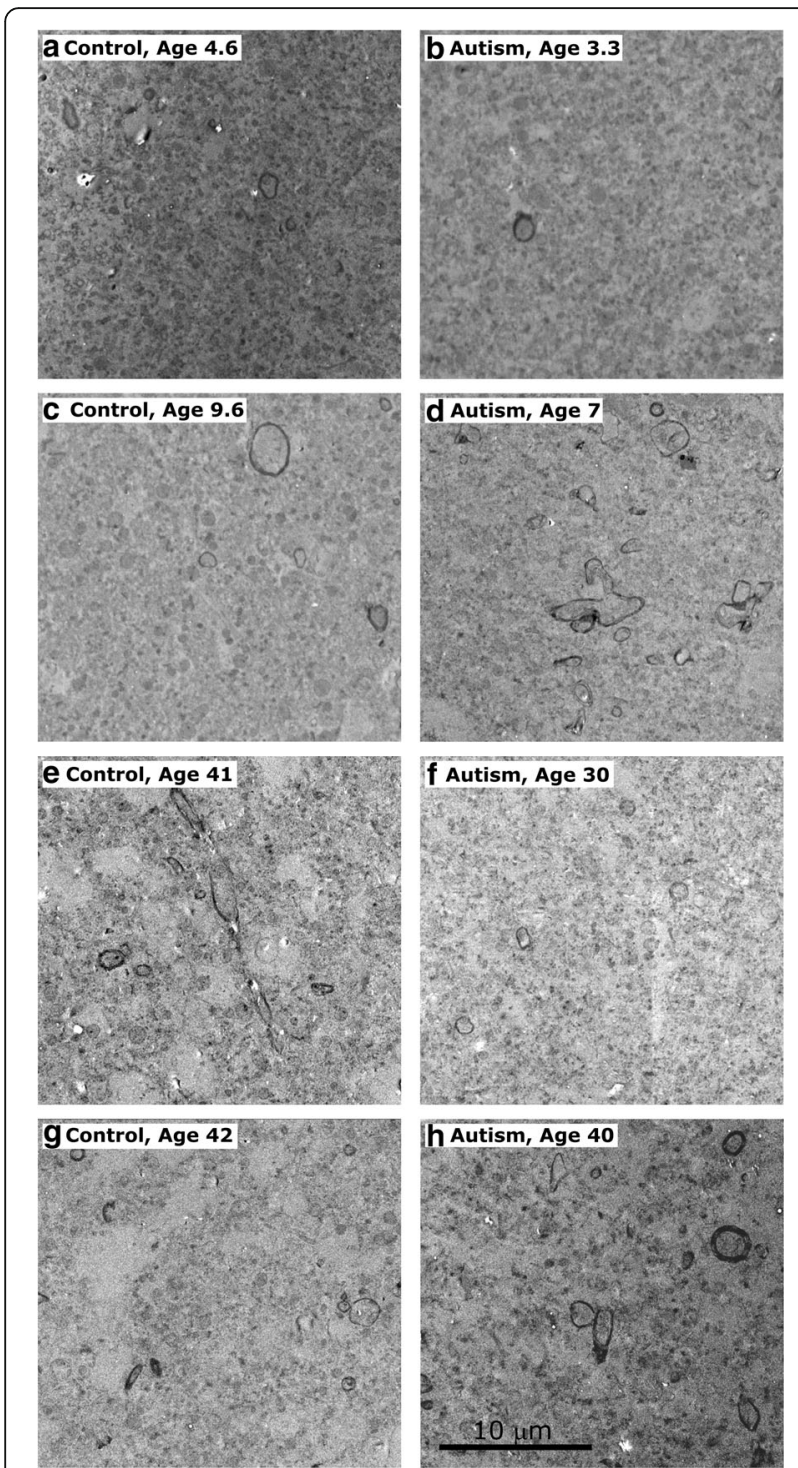

Fig. 3 Myelinated axons in LPFC layer 1 analyzed at the electron microscope level in young children [cases 451 (a), 4021 (b)], older children [cases M3835 M (c), AN 03321 (d)], and adults [cases 5353 (e), $5173(\mathbf{f}), 4981(\mathbf{g}), 6232$ (h)] illustrate described trends. In younger children, who overall had fewer myelinated axons, there is still visible reduction in density of myelinated axons in individuals with autism (b). In older children there was a clear increase in the density of myelinated axons in autistic children (d), representing the variability inherent in autism, and multiple branching axons can be seen within the field. In adults, there was a decrease in proportion of small axons in individuals with autism with no visible change in overall axon density $(p=0.390)(\mathbf{f}, \mathbf{h})$. Scale bar in (h) applies to all panels

$\mathrm{CB}$, and $\mathrm{CR}$. In humans, neurons in layer 1 are overwhelmingly inhibitory: the vast majority of neurons in layer 1 express GABA [55] and exhibit inhibitory activity [106]. We characterized age-related changes in the density and distribution of neurons in layer 1 in neurotypical children and adults and compared them with trends seen in individuals with autism to identify whether changes in neuronal populations could be additional sources of excitatory-inhibitory imbalance in autism (Fig. 4).

\section{Normative development of neurons in layer 1}

We measured neuron density in layer 1 in neurotypically developing children and adults in Nissl-stained tissue. In order to ensure accuracy, tissue was oversampled to account for the low density and anisotropic distribution of cells in layer 1 . Overall neuron density in layer 1 in adults was $7059 \pm 1242$ cells $/ \mathrm{mm}^{3}$. Neuron density declined from childhood to adulthood (Fig. 4a, b), likely due to the increase in neuropil volume associated with branching and myelination during development. There was a trend towards an increase in neuron density in layer 1 with age in older adults (Fig. 4e), potentially relating to the changes in axon pruning with age described above. In children, we saw significant variability in the neuron density in layer 1 , in line with reported heterogeneity in the maturation of the cortex [127]. Cells occupied $10.6 \pm 3.8 \%$ of the area of layer 1 in children; this increased moderately to $12.1 \pm 5.4 \%$ in adults (Fig. 4 f).

\section{Inhibitory neurons in layer 1 during neurotypical development}

In order to further study this population of inhibitory neurons, we labeled tissue with the three calcium-binding proteins (PV, CB, and $\mathrm{CR}$ ). The most prevalent inhibitory interneuron subtype in layer 1 in neurotypically developing individuals were $\mathrm{CR}+$ interneurons (54-94\% of labeled neurons) (Fig. 4l), which, in the upper cortical layers, primarily serve a disinhibitory role through selective targeting of $\mathrm{CB}+$ interneurons $[29,86]$. This is in line with previously reported findings in layer 1 in the adult human and non-human primate cortex, which has been shown to predominately contain CR neurons, including remnants of the Cajal-Retzius cell population [40, 81, 82]. There were also few calbindin-expressing neurons (mean $=194 \pm 127$ cells $/ \mathrm{mm}^{3}$ ), which did not vary significantly with age. In children, CR neurons had a mean density of $1270 \pm 475$ cells $/ \mathrm{mm}^{3}$, which increased in adults to $1437 \pm 902$ cells/ $\mathrm{mm}^{3}$ (Fig. 4h). We separately analyzed younger (37-47 years old) and older adults (58-67 years old) to identify possible age-related changes in $\mathrm{CR}$ neuron density. In younger adults CR neuron density in layer 1 was $1009 \pm$ $488 \mathrm{cells} / \mathrm{mm}^{3}$, while preliminary data from two older adults suggests an increase in CR neuron density (mean = $2508 \pm 847$ cells $/ \mathrm{mm}^{3}, p=0.006$ ). Quantification of additional cases with ages over 60 years would be necessary to confirm this change. Of note, the majority of neurons in layer 1 in adults were not labeled by calcium-binding proteins (69-94\%) (Fig. 4m). 


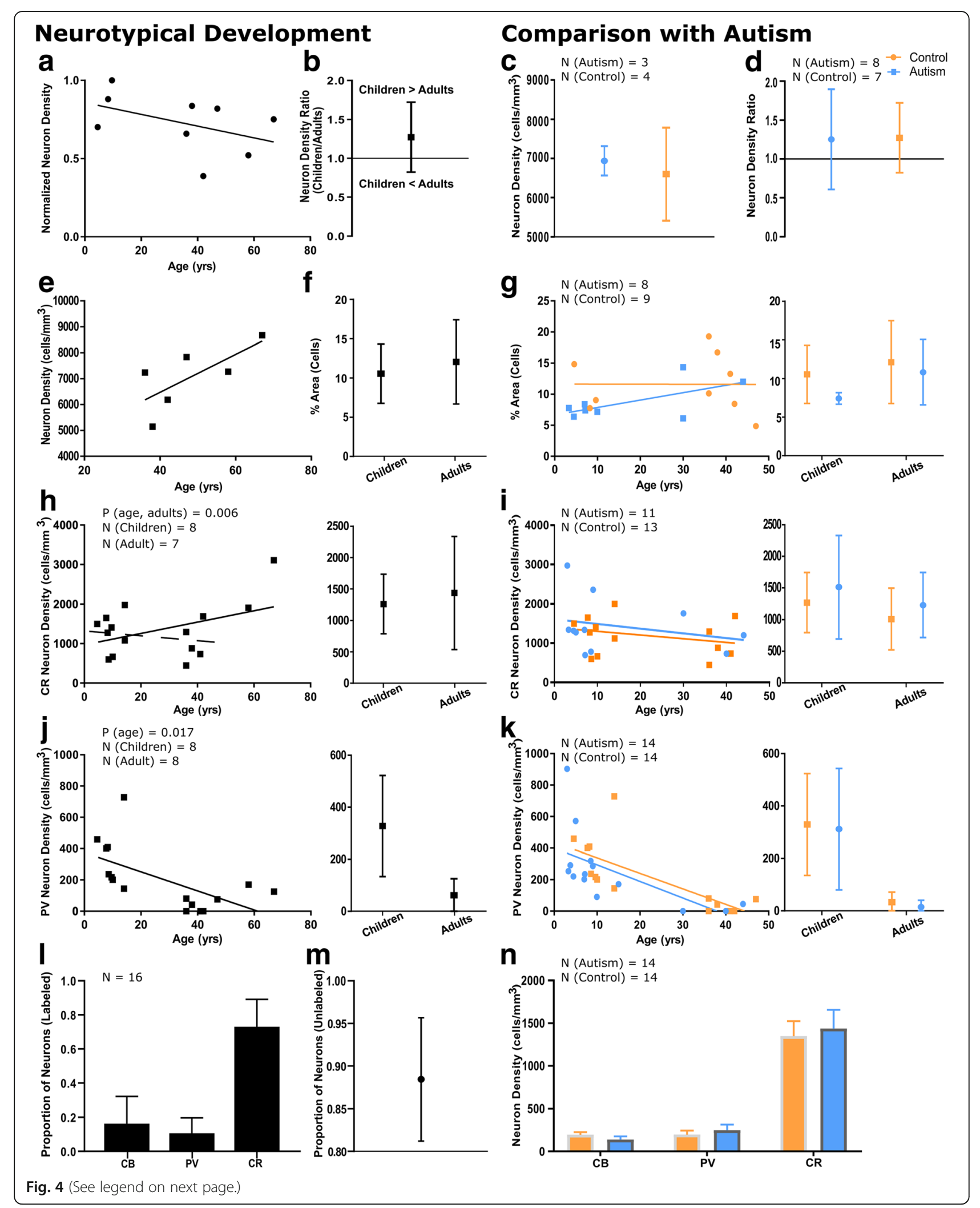


(See figure on previous page.)

Fig. 4 Neuronal populations in LPFC layer 1 change in parallel between children and adults with and without autism. a There was a trend towards a reduction in neuron density between neurotypical children and adults. Neuron density values were normalized such that the highest density has a value of 1. Cases used in this analysis: 451, 4337, M3835 M, 6004, 4981, HAW, HAY, HCD, HCF. b There was a reduction in neuron density between typical children and adults, with a ratio of mean neuron density in children/adults of approximately 1.27. c Mean neuron density in adults with and without autism (mean control $=6602 \pm 1186 \mathrm{cells} / \mathrm{mm}^{3}$, mean autism $=6939 \pm 217$ neurons $/ \mathrm{mm}^{3}$ ) was not statistically different. d The decrease in neuron density from childhood to adulthood was not different between groups. e There was a trend towards an increase in neuron density with age between young and older adults. $\mathbf{f}$ The area fraction containing cells increased slightly in neurotypical development, likely representing simultaneous dilution of neurons within the neuropil and expansion of glial cell populations with age. $\mathbf{g}$ There was no difference between the percent surface area occupied by cells between autistic and neurotypical groups. $\mathbf{h}$ CR neuron density increased between children and adults. In this sample, this trend was primarily driven by high density of CR neurons in older adults. The solid line shows the overall trend towards an increase in CR neuron density with age, the dotted line shows the decrease in CR neuron density when comparing children and young adults $\mathbf{i}$ The trend towards a decrease in CR neuron density in layer 1 was similar in neurotypical individuals and individuals with autism. j PV neurons were present in layer 1 in neurotypical children; their density decreased significantly in adults, and in many adult cases these neurons were not found. $\mathbf{k}$ PV neurons were prominent in layer 1 of non-autistic (mean $=329 \pm 69$ neurons $/ \mathrm{mm}^{3}$ ) and autistic children (mean $=$ $312 \pm 231$ neurons $/ \mathrm{mm}^{3}$ ), and their density decreased significantly in adults (mean control $=33 \pm 39 \mathrm{cells} / \mathrm{mm}^{3}$, mean autism $=15 \pm 26$ neurons/ $\left.\mathrm{mm}^{3}, p=0.006\right)$. I We calculated the relative proportions of the three inhibitory interneuron subtypes in layer 1 . CR was the most prominent inhibitory interneuron subtype, labeling $73.07 \pm 16.07 \%$ of labeled neurons. CB was expressed by $16.32 \pm 15.81 \%$ of labeled inhibitory neurons, while PV was the least prominent interneuron subtype labeling only $10.60 \pm 9.10 \%$ of labeled interneurons in layer 1 . $\mathbf{m} 88.44 \pm 7.24 \%$ of neurons were not labeled with calcium binding proteins in layer 1. $\mathbf{n}$ We calculated mean inhibitory neuron densities, including cases of all ages. CRimmunoreactive neurons were the densest neuron population (mean control $=1385 \pm 696$ neurons $/ \mathrm{mm}^{3}$, mean autism $=1436 \pm 733$ neurons/ $\mathrm{mm}^{3}$ ), followed by CB- (mean control $=194 \pm 127$ neurons $/ \mathrm{mm}^{3}$, mean autism $=138 \pm 124$ neurons $/ \mathrm{mm}^{3}$ ) and PV- neurons (mean control $=220 \pm$ 215 neurons $/ \mathrm{mm}^{3}$, mean autism $=248 \pm 239$ neurons $/ \mathrm{mm}^{3}$ ). There were no significant differences between groups. Autism cases AN 03345, 1182, AN 04682, and B-6677 and control case HCF were not included in CR quantitative analyses due to inconsistent staining. Autism case B-6677 was not included in PV quantitative analyses due to inconsistent staining. Left panels in $\mathbf{~ g - k ~ p r e s e n t ~ d e v e l o p m e n t a l ~ t r e n d s ~ o n ~ a ~ c a s e - b y - c a s e ~ b a s i s , ~}$ and right panels show mean \pm SD for children and adults

\section{PV-immunolabeled neurons in layer 1 of children and adolescents}

Our results show for the first time to the best of our knowledge that neurotypically developing children and adolescents have a population of PV neurons in layer 1 in LPFC $\left(\right.$ mean $=329 \pm 194$ neurons $\left./ \mathrm{mm}^{3}\right)$ (Fig. 4j). Previous studies have identified PV-expressing Cajal-Retzius and non-Cajal-Retzius neurons in layer 1 of prenatal and newborn humans [30, 121]: the neurons identified in this study may be members of these neuronal populations, which persist later in development than previously believed. Further studies are needed to identify the functional role of these PV-expressing neurons of layer 1, which may or may not serve the same strongly-inhibitory role of the PV-expressing neurons of the deeper cortical layers.

There was a significant age-related reduction in the density of PV interneurons in adults, with many cases having no detectable PV neurons in layer 1 (mean $=62$ \pm 22 neurons $/ \mathrm{mm}^{3}, p=0.017$ ) (Fig. 4j). Younger adults had a mean PV neuron density of $33 \pm 39$ cells $/ \mathrm{mm}^{3}$, which increased to $148 \pm 32$ cells $/ \mathrm{mm}^{3}$ in older adults, based on preliminary data.

\section{No changes in neurons in layer 1 in autism}

There was no significant difference between control and autism groups in overall neuron density in layer 1 in adults (mean Autism $=6939 \pm 217$ neurons $/ \mathrm{mm}^{3}$; mean Control $=6602 \pm 1186$ cells $/ \mathrm{mm}^{3}, p=0.453$ ) (Fig. 4c). The decline in neuron density associated with age (Fig. 4d) and the percent surface area occupied by cells (Fig. $4 \mathrm{~g}$ ) were also similar between groups. Previous reports have identified as much as a $30 \%$ increase in neuronal density in the prefrontal cortex in autism [3, 16]; our findings suggest that these increases are not due to changes in layer 1 .

We assessed the densities of the three interneuron subtypes in layer 1 of LPFC in individuals with autism and found no differences from controls $[\mathrm{p}(\mathrm{CR}$, Autism vs Control $)=0.470, \mathrm{p}(\mathrm{PV}$, Autism vs Control $)=0.828$, $\mathrm{p}(\mathrm{CB}$, Autism vs Control $)=0.711$ ] (Fig. 4i, k, n). This suggests that the changes reported above in myelinated axon structure in autism are not due to changes in the cellular populations intrinsic to layer 1, but instead are due to changes in branching and maturation of local networks or incoming pathways.

\section{Cross-validation of layer 1 features through systematic comparison of frontal cortices in adult humans and in a non-human primate animal model}

In order to confirm that our results were not influenced by post-mortem factors associated with the acquisition and processing of human LPFC tissue, we cross-validated our findings with a qualitative assessment of the cellular populations and myelination in another prefrontal region of the human brain, the ACC, and in optimally-fixed rhesus macaque tissue. A comparison of the patterns of immunohistochemical labeling of immersion-fixed brain tissue with a post-mortem delay and ideally-fixed, perfused brain tissue has suggested that the detection of some 
calcium-binding proteins is susceptible to post-mortem changes [50]. As both the gray and white matter of the non-human primate have significant structural and organizational similarity to that of the human [131], we used perfused tissue from rhesus macaque monkeys to identify the key structural characteristics of the normal layer 1 and to confirm that our findings in the human were not attributable to changes in immunoreactivity due to post-mortem factors. We therefore compared the structure of layer 1 in LPFC to ACC in both species and identified conserved and altered features of layer 1 in these structurally distinct areas (Figs. 5, 6 and 7). Images of representative neurons and glial cells are summarized in Fig. 8. Our comparisons, summarized below, highlight the high biological and statistical significance of the reported changes in both typical and atypical postnatal development of layer 1 in the human LPFC.

There was a moderate density of neurons in layer 1 in both areas (Fig. 5a, e; Fig. 6a, e; 7a, c). A subpopulation of layer 1 neurons was labeled with GABA or GAD67, indicating that they were inhibitory (Figs. 5f-g, 6f-g). Few neurons were labeled by $\mathrm{CR}$, which indicates that they were either inhibitory or non-GABAergic Cajal-Retzius neurons. CB- and PV-expressing inhibitory neurons were scarce in layer 1 of adult primates, although some cell processes within layer 1 expressed these markers strongly (Figs. 5h-j, 6h-j, 7e-g). Qualitative observations from the non-human primate were in line with qualitative and quantitative findings in human subjects. These observations suggest that post-mortem factors did not significantly alter the conclusions of our immunohistochemical analysis.

Varied populations of glia were present in layer 1 (Figs. 5k-l, 6k-l, 7h-j). Astrocytes, which participate in the regulation of neuronal signaling [105], were highly immunoreactive for GFAP, a glial structural protein associated with astrocyte activation (Figs. 5l, 6l), but did not express EAAT2, an excitatory amino acid transporter responsible for the reuptake of glutamate (Fig. 7j). We furthermore identified interlaminar astrocytes, which are typical of layer 1 in the primate brain and extend processes though layers 2 and 3 [19, $21,79,80]$ and marginal astrocytes on the pial border that extended processes towards layer 2 (Fig. 7i). The unique structure and function of astrocytes in layer 1 likely affects the regulation of signaling in this layer and merits future consideration.

There was a dense, superficial plexus of myelinated axons in layer 1 . Myelinated axons were seen penetrating layer 1 to join this plexus (Figs. 5b, 6b, 7b, d), supporting the assertion that some myelinated axons within layer 1 originate outside of this layer. This plexus was denser in LPFC than in ACC (Figs. 5b, 6b, 7b, d), reflecting an overall difference in myelination between these areas. The myelinated plexus in LPFC furthermore contained a higher density of PV-positive axons (Figs. 5j, 6j, $7 \mathrm{~g}$ ), deriving either from local interneurons or thalamocortical pathways [47]. Neuropil in layer 1 was densely labeled by $\alpha$-CamKII (Figs. 5d, 6d), a marker of synaptic plasticity [75]: high levels of this protein in layer 1 suggests that networks within layer 1 in these cortices are remarkably plastic. Differences between the structure of axon networks in the granular LPFC and limbic ACC despite relative homogeneity in the cellular populations of layer 1 supports our conclusion that the plastic and variable axonal networks within layer 1 could be a target of dysfunction in autism.

\section{Discussion}

We present evidence of postnatal changes in the balance of excitation-inhibition in the maturing prefrontal cortex throughout typical development and in autism, using a large cohort of human subjects at a variety of postnatal ages. Our findings reveal specific changes in the structure of pathways and cellular populations within layer 1 of the LPFC through typical development. We also present evidence suggesting that atypical, age-associated changes in the organization and relationship between pathways and cellular populations in layer 1 of the LPFC may underlie the dysfunctional balance of excitation-inhibition in the maturing prefrontal cortex in autism.

In typical postnatal development, the density of myelinated axons in layer 1 of the prefrontal cortex increased with age, in line with previous studies on the maturation of white matter pathways [74, 89]. Specifically, in LPFC, the relative proportion of thin myelinated axons in layer 1 of adults was significantly higher than what has been previously described in the white matter, where thin axons represented $36 \%$ of the myelinated axon population [129, 131, 133]. This supports our understanding that axons enter the cortex and quickly branch, resulting in an overrepresentation of thin axons in superficial cortical layers. The increased density of thin axons in layer 1 was accompanied by an increase in the volume of neuropil, including an increase in unmyelinated axon density, estimated by the gray level index, and a decrease in neuron density in adults. We noted both an increase in neuron density and a decrease in the proportion of thin axons in two older adults (ages 58 and 67), suggesting that reductive changes in neuropil structure, potentially due to pruning, may be associated with normal aging.

Surprisingly, a significant proportion of neurons in layer 1 did not express the calcium-binding proteins that are typically used as markers of inhibitory interneurons in the cortex of primates, even though close to $90 \%$ of neurons in layer 1 are GABAergic [41, 42, 54-56]. 

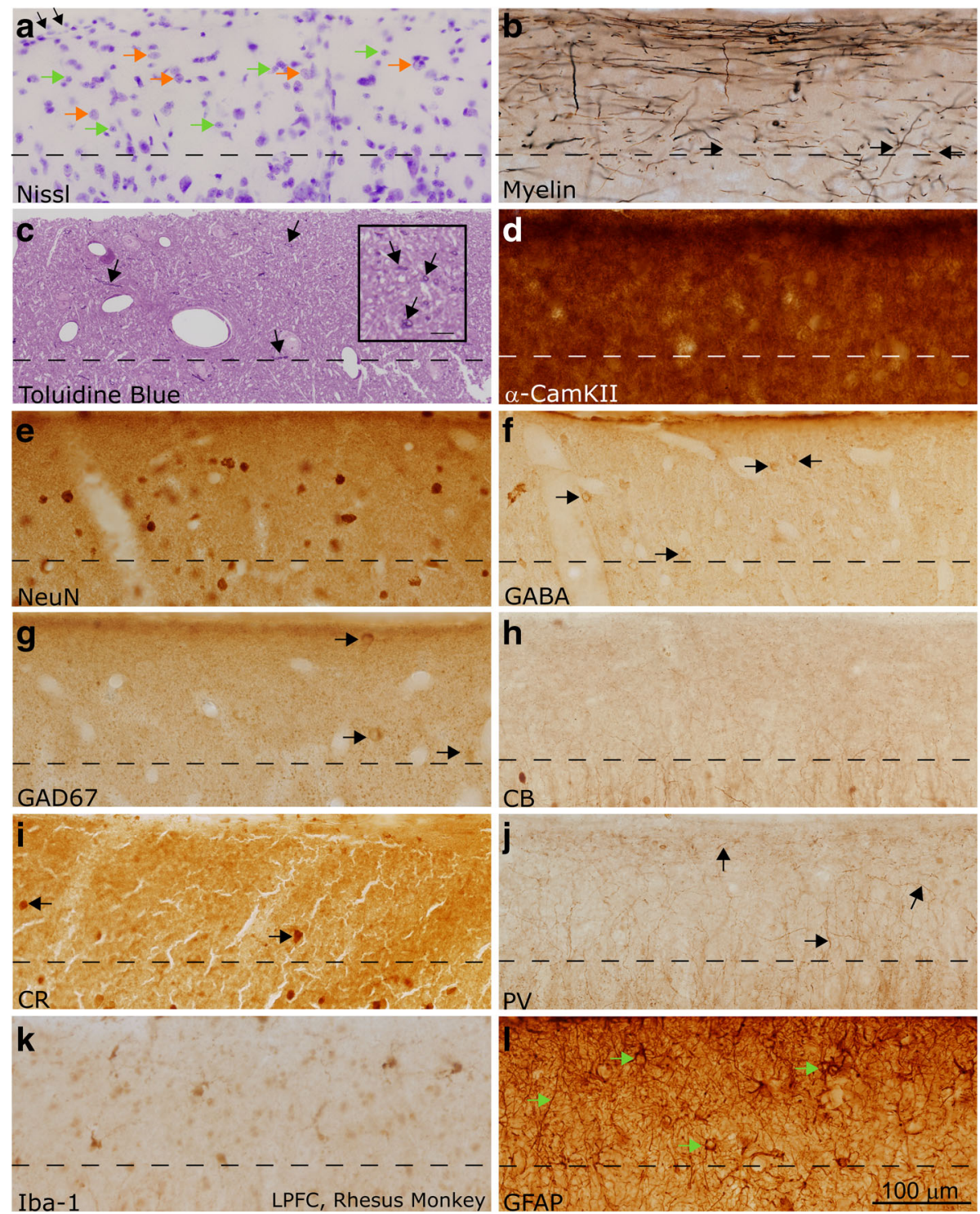

Fig. 5 Layer 1 in the LPFC of the adult non-human primate (rhesus macaque). a Section labeled with Nissl showed a moderate density of neurons and glia, mainly astrocytes, in layer 1 (examples of neurons are marked with orange arrows, glial cells are marked with green arrows). Superficially, the glia limitans was visible as a dense band of astrocytes (shown with black arrows). b Myelin (Gallyas) stain showed the dense band of myelinated axons within superficial layer 1. Myelinated axons were seen penetrating layer 1 with diverse trajectories prior to joining the myelinated superficial plexus (black arrows). These axons came from long-range pathways or local interneurons. c Toluidine blue labeling of a $1 \mu \mathrm{m}$ thick section from osmicated tissue revealed Nissl-stained cells and their processes, including profiles of myelinated axons (shown with black arrows). There was a higher density of thin axon profiles in superficial layer 1, in line with myelin stain shown in (b). Scale bar in inset measures $10 \mu \mathrm{m}$. $\mathbf{d}$ a-CamKIl is a marker of synaptic turnover, and is used to identify areas of high plasticity. It labeled a dense population of processes in layer 1. e NeuN labels most neuronal nuclei, and in our material all layer 1 neurons that were labeled with Nissl were also labeled with NeuN (not shown). NeuN labeling clearly showed that the density of labeled neurons in layer 1 was low, and the majority of the cellular density in layer 1, as seen in (a), was not due to neurons but instead could be attributed to glia. $\mathbf{f}-\mathbf{g}$ GABA (f) and GAD67 (g) label inhibitory neurons in the cortex (labeled with black arrows). There was a low density of labeling in both sections. Comparison with sections stained with NeuN (c) suggested that many neurons in layer 1 did not express GABA and its synthesizing enzymes strongly. h-j CB, CR, and PV labeled subpopulations of inhibitory interneurons. CB (h) and PV (j) did not label cell bodies in layer 1 in the adult non-human primate, while CR (i) labeled few cell bodies (shown with black arrows). PV (j) labeled a population of axons which joined the superficial plexus, and may represent either thalamocortical axons or axons of local inhibitory interneurons (shown with black arrows). CB (h) and CR (i) also labeled few axons in layer 1, but were not as visible as the PV-labeled processes. k Microglia with various morphologies, labeled with Iba-1, could be seen in layer 1. I GFAP labeled the cell bodies and dense processes of astrocytes within layer 1 (shown with green arrows). Images were acquired such that the top edge of the images underlie the pia. Dotted lines indicate the border with layer 2 in all panels. Calibration bar in (I) applies to all panels 

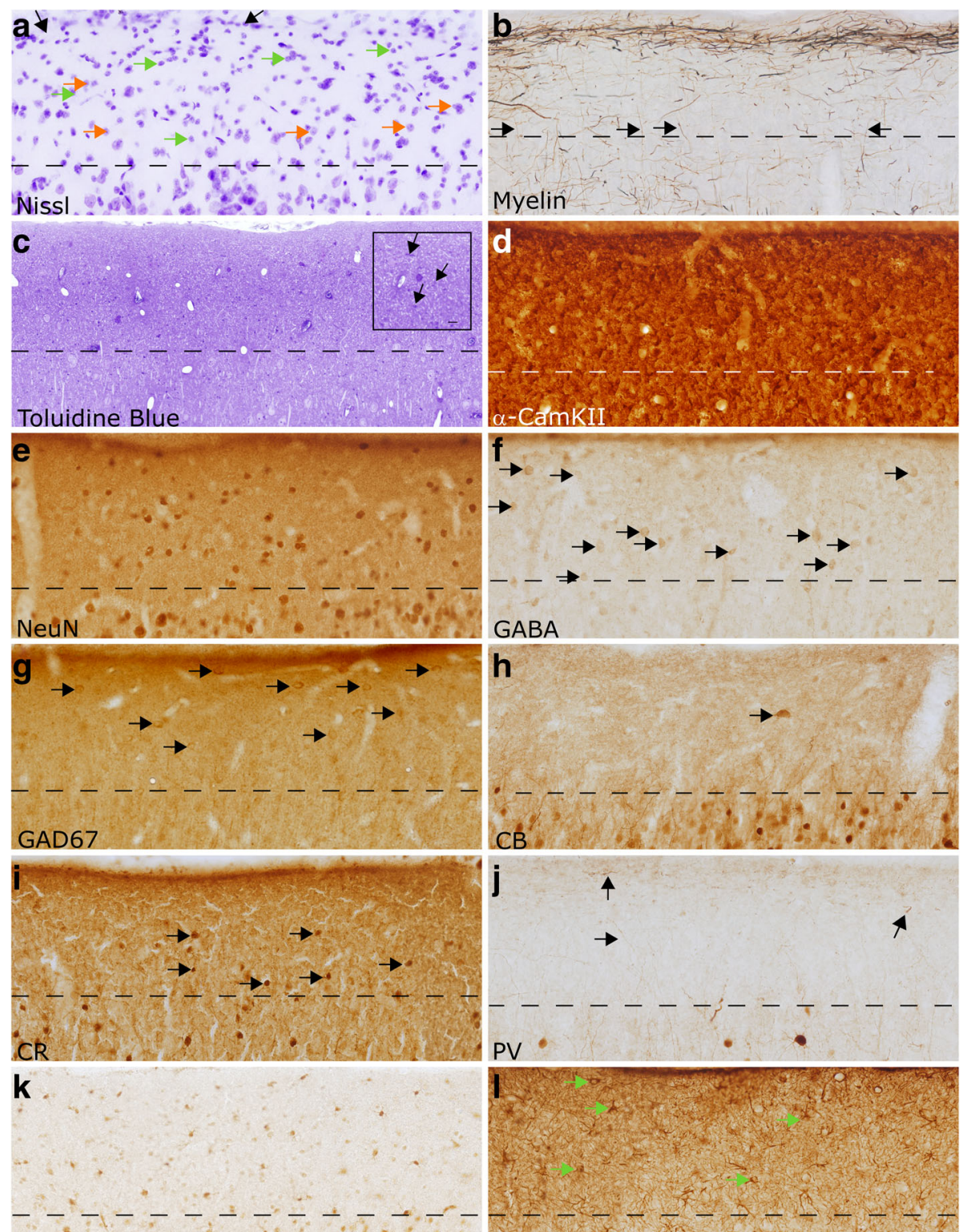

Iba-1 $\quad$ ACC, Rhesus Monkey GFAP

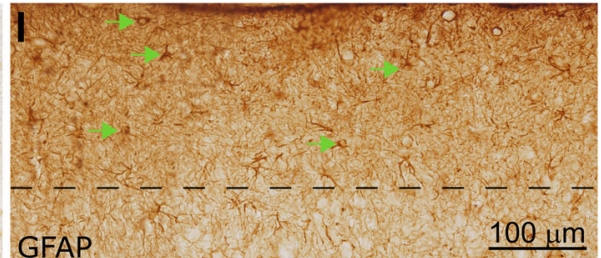

Fig. 6 Layer 1 in the ACC of the adult non-human primate (rhesus macaque). a Section labeled with Nissl showed a moderate density of neurons and glia, mainly astrocytes, in layer 1 (examples of neurons are marked with orange arrows, glial cells are marked with green arrows). Superficially, the glia limitans was visible as a dense band of astrocytes (shown with black arrows). b Myelin (Gallyas) stain showed the relatively light band of myelinated axons within superficial layer 1 in ACC. This band was lighter than that seen in LPFC, following overall trends in myelination between those areas. Black arrows show axons entering layer 1 from layer 2. c Toluidine blue labeling of a $1 \mu \mathrm{m}$ thick section from osmicated tissue revealed Nissl-stained cells and their processes, including profiles of myelinated axons (shown with black arrows in inset). There was a low density of axon profiles in superficial layer 1, in line with the light myelin stain shown in (b). Scale bar in inset measures $10 \mu \mathrm{m}$. $\mathbf{d}$ a-CamKIl is a marker of synaptic plasticity. It labeled a dense population of processes in layer 1. e NeuN labeled all layer 1 neurons that were labeled with Nissl (not shown). NeuN labeling clearly showed that the density of labeled neurons in layer 1 was low, and the majority of the cellular density in layer 1 , as seen in (a), was not due to neurons but instead could be attributed to glia. $\mathbf{f - g}$ GABA (f) and GAD67 (g) labeled inhibitory neurons in the cortex (labeled with black arrows). There was a moderate density of labeling in both sections, comparable to what was seen in the NeuN stain (c). $\mathbf{h}-\mathbf{j}$ $\mathrm{CB}, \mathrm{CR}$, and PV labeled subpopulations of inhibitory interneurons. CB (h) and PV (j) labeled primarily neuron processes in layer 1 in the adult nonhuman primate, while CR (i) labeled few cell bodies (shown with black arrows). Black arrows in (j) show PV-labeled axons. k Microglia with various morphologies, labeled with Iba-1, could be seen in layer 1. I GFAP labeled the cell bodies and dense processes of astrocytes within layer 1 (shown with green arrows). Images were acquired such that the top edge of the images underlie the pia. Dotted lines indicate the border with layer 2 in all panels. Calibration bar in (I) applies to all panels 

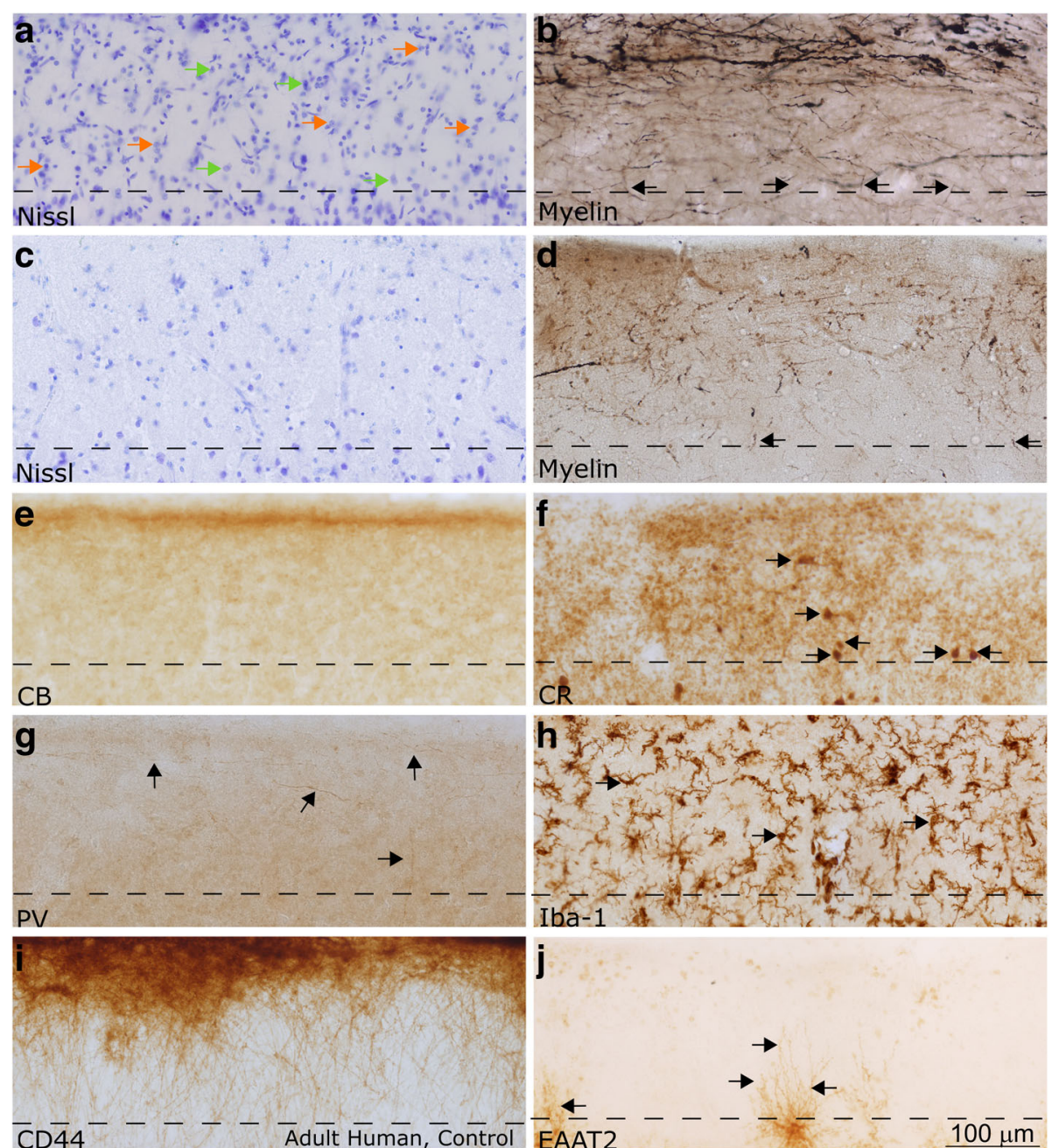

Fig. 7 Layer 1 in the LPFC and ACC of the adult human. All images in this figure were acquired from neurotypical adult cases (HCD, HCF, HAW, HAY). a-d Nissl (a) and myelin (b) in the LPFC showed stark differences from ACC (c-d). ACC had a thicker layer 1, and reduced density and thickness of myelinated axons in layer 1. Permeating blood vessels and endothelial cells were also visible in the Nissl-labeled section from LPFC layer 1 in (a). Examples of neurons in (a) are marked with orange arrows, glial cells are marked with green arrows. Myelin (Gallyas) stained tissue (b, d) showed a dense plexus of myelinated axons in superficial layer 1, consistent with observations in the non-human primate. While the majority of axons were horizontal, some also had diagonal trajectories, consistent with axons from incoming pathways (black arrows). e-g CB, CR, and PV labeled inhibitory interneuron classes. CB (e) labeled scant processes in layer 1 (labeled with black arrows), while CR (f) labeled a low density of neurons and few processes in layer 1 (labeled with black arrows). PV (g) labeled processes that joined the plexus of axons in superficial layer 1 (labeled with black arrows). $\mathbf{h}$ lba-1 labeled microglia within the cortex, including layer 1 (examples of microglia are labeled with black arrows). Superficial microglia extended processes mostly parallel to the pial surface, while microglia deeper in layer 1 had processes oriented in multiple directions. i CD44 labeled interlaminar astrocytes, which sat on the pial surface and sent processes towards layer 2. These astrocytes are exclusive to higher-order primates. $\mathbf{j}$ Astrocytes labeled with excitatory amino acid transporter (EAAT2) were not present in layer 1; however, the processes of labeled astrocytes from layer 2 penetrated into the deep part of layer 1 (labeled with black arrows). Images were acquired such that the top edge of the images underlie the pia. Dotted lines indicate the border with layer 2 in all panels. Calibration bar in (j) applies to all panels

Similar staining patterns in human and optimally-fixed rhesus macaque brain tissue corroborated this observation. These findings suggest that there are potential differences in the origin and physiological characteristics of interneurons in layer 1 when compared to other cortical layers, which may influence the regulation of GABAergic signaling in layer $1[28,102]$. The emerging complexity of this layer in our study is in line with recent work that has led to the identification of novel neuronal types in layer 1 of humans [11]. The differences that we have identified in the expression of calcium-binding proteins between childhood and adulthood further suggest that the cellular composition of layer 1 changes significantly during postnatal development. We detected a significant 


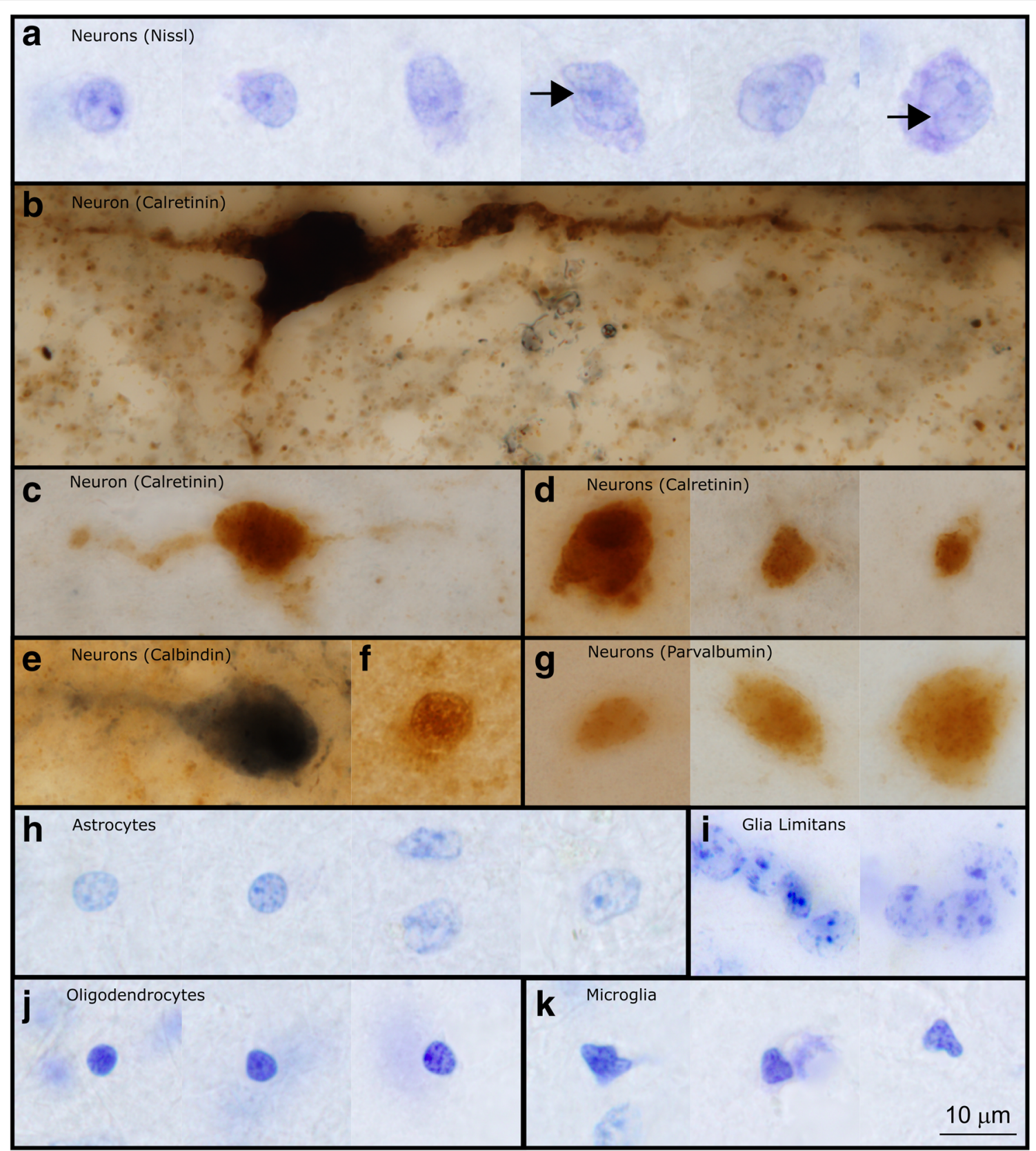

Fig. 8 High magnification images of representative neurons and glia in layer 1 of the LPFC in the human brain. a Neurons in layer 1 had a broad range of sizes. In Nissl preparations it was possible to see the nucleus, nucleolus, and a rim of cytoplasm around labeled neurons. In larger neurons it was possible to observe folding of the nucleus (shown with black arrows in figure) (Case HCD). b This large, subpial neuron labeled with CR is likely a Cajal-Retzius cell. This image shows its large cell body and horizontal extensions, along with a descending process which is typical of Cajal-Retzius cells (Case AN4722). c-d CR labeled neurons of multiple sizes and shapes were found in layer 1 of the human cerebral cortex. CR-labeled neurons in layer 1 had multiple orientations within layer 1, including horizontally oriented cells (c) and cells with more typical interneuron morphology (d). e-f CB-labeled neurons were also diverse in shape and size. These neurons may also be oriented horizontally (e) (Case 4722, Case 3835). g Representative PV-labeled neurons in layer 1 (Case 4337). h-i Astrocytes in layer 1 had multiple morphologies. The astrocytes of the glial limitans were compact and formed tight groups (i) (Case HCD). $\mathbf{j}$ Three oligodendrocytes in human layer 1 (Case HCD). Oligodendrocytes in human tissue had varied staining characteristics: they could be darkly labeled, as seen in the first two images, or lightly labeled, as shown in the third image. k Microglia in layer 1 had irregularly shaped nuclei, distinguishing them from darkly stained oligodendrocytes (Case HCD). Calibration bar in (k) applies to all panels 
population of PV-immunoreactive neurons in LPFC layer 1 of neurotypical children and adolescents, previously seen only pre- and peri-natally [30, 121]. The density of layer $1 \mathrm{PV}$-immunoreactive neurons decreased with age to negligible numbers in adults, suggesting developmental changes in the calcium dynamics, and therefore in synaptic speed and strength, of inhibitory neurons in layer 1 during development. This could have an effect on the processing of incoming feedback or neuromodulatory signals in childhood and adulthood, leading to differences in the balance of excitation-inhibition in the cortex.

We found significant evidence of disorganization of axon networks within layer 1 in individuals with autism. There was increased heterogeneity in the trajectories and the proportion of myelinated axons that were thin in children with autism compared with controls. While there was an increase in the ratio of thin myelinated axons in layer 1 in neurotypical adults, thin axon ratios did not change with age in autism, leading to a significantly lower proportion of thin myelinated axons in adults with autism compared to the control group. This suggests that layer 1 thin axon networks in adults with autism remained on average at similar levels as immature, less myelinated networks in childhood, in line with previous work that has shown increased axon branching at the border of the gray and white matter below interlinked medial and lateral prefrontal cortices in these and other cases [35, 111, 129-131, 133]. Increased axon branching and a decrease in the proportion of thin myelinated axons may be due to an increase in the density of unmyelinated axons, which we could not directly assess in this study. Future studies would need to assess changes in the qualities of the neuropil through development and in autism to confirm this hypothesis.

Alterations in axon structure may have broader implications for the efficacy of signal transmission in frontal cortical networks of individuals with autism. In particular, changes in axon caliber and the relative ratios of myelinated axon sizes directly influence the strength and persistence of the action potential generated by projection neurons, as well as their firing rate $[58,96]$, which play a key role in sustained activity during working memory and attentional tasks in LPFC $[22,65,95,99]$. These, in turn, can underlie changes in the spread of activity and oscillations $[12,59,67,68,73]$, and may be linked with changes in the expression of neuronal ion channels and regulatory proteins in individuals with autism [25, 57, 94, 98]. Genes associated with autism, such as UBE3B and ZNF18, are transcriptionally regulated by membrane depolarization and are involved in experience-dependent learning and synaptic plasticity [34]. These processes are also regulated by reelin in the cortex, and especially layer 1 , throughout the lifespan in primates $[72,83]$. Importantly, changes in axons in children and adults in autism were not accompanied by alterations in the overall density of neurons nor in the density of labeled inhibitory interneuron subclasses in layer 1 of LPFC, suggesting that these changes are specifically isolated to axon networks.

Previous examination of LPFC has revealed moderate changes in the expression of reelin in layer 1 of some individuals with autism [115]. Reelin, which is distributed throughout the soma and dendrites of most neurons in layer 1 [83], is secreted by Cajal-Retzius cells during prenatal development. Reelin has also been implicated in the regulation of synaptic plasticity and learning in the postnatal brain and is involved in the pathogenesis of neuropsychiatric disorders (reviewed in [37-39, 72]). Importantly, a study of the human temporal lobe found no change in the density of reelin-expressing neurons in autism: consistent with the findings reported here, this study further identified no change in neuron density in layer 1 in autism [14]. Taken together, our findings and previous reports suggest that reduction in the expression of reelin may be due to changes in protein processing or intracellular distribution within the cell population of layer 1 that can contribute to cortical patterning defects and postnatal changes in network structure in autism.

Other protein factors with altered expression in LPFC layer 1 in autism include chemokine ligand 14 (CXCL14) and neuron-derived neurotrophic factor (NDNF). CXCL14, an inflammatory cytokine, is involved in the regulation of myelination; reduction in the expression of this cytokine results in a reduction in myelination [4, $119]$, which is consistent with our findings of reduced myelination and increased branching in prefrontal cortices of adults with autism [129, 131, 133]. NDNF is mainly expressed by Cajal-Retzius cells of layer 1 , and promotes the growth and development of neuronal cell bodies and neurite outgrowth [70, 106]. Disruption of any of these factors may adversely impact the development of the cortical column in LPFC, and could specifically underlie the atypical organization of circuits within layer 1 of LPFC in autism.

Placing our findings within the context of previous studies that have reported neuropathological and molecular changes in prefrontal cortices in autism offers additional clues about mechanisms that may underlie specific disruption of LPFC networks. Major feedback pathways that target layer 1 of LPFC and may contribute to the observed axon disorganization include pathways from high-order thalamic nuclei $[60,128]$ and, to a lesser extent, the amygdala [48], regions that are consistently affected in autism $[8,9,51,76,107-109,117,118$, 120]. Based on extensive experiments on a large cohort of subjects we have proposed that disruption of networks in autism depends on the time of the insult during prenatal development of cortical pathways [129]. A general, consistent theme that emerges from our 
findings is that feedback, short-range pathways from the deep layers of limbic cortices that target superficial layers of eulaminate cortices, which develop relatively early but mature late postnatally, are susceptible to disruption and are affected in autism [45, 129-131, 133]. We previously reported that in the neighboring ACC there is an increase in the expression of GAP-43 [129], a growth axon protein that is antagonistic to myelin basic protein [64] and promotes branching and shedding of myelin of axons. The ACC in primates is a major contributor of robust feedback pathways that terminate in the superficial layers of LPFC, including layer $1[18,20$, $69,85,91,131]$. It is therefore conceivable that the observed axon pathology in layer 1 of LPFC may be due to disruption in this short-range feedback network linking ACC and LPFC [45]. This hypothesis is also supported by the oft reported over-connectivity of local frontal networks in autism [24, 113].

In conclusion, we systematically examined layer 1 of LPFC in individuals with and without autism at high resolution. We described the typical postnatal development and organization of axon circuits and local interneurons. Study of excitatory and inhibitory circuit components in parallel provided a novel framework that facilitated identification of pathological changes within cortical networks in autism. We found significant changes in the structure and organization of myelinated axons in LPFC layer 1 in individuals with autism, with important implications for the balance of excitation-inhibition and local cortical information processing. Our findings highlight feedback pathways in LPFC as an especially vulnerable node that underlies autism pathophysiology. Finally, our synthesis of the new findings with previous studies provide important clues that can help link the atypical development of frontal networks in autism with key molecular mechanisms and factors, whose interactions during development will need to be elucidated in future studies.

\section{Acknowledgements}

We gratefully acknowledge donors and their families, the Autism Tissue Program, the Harvard Brain Tissue Resource Center, the Institute for Basic Research in Developmental Disabilities, the University of Maryland Brain and Tissue Bank, the National Disease Research Interchange (NDRI), and Anatomy Gifts Registry for providing post-mortem human brain tissue. We thank Tara McHugh and Maalavika Ragunathan for technical assistance, Marcia Feinberg for assistance with electron microscopy, and Dr. Helen Barbas for the use of archival processed non-human primate tissue and useful discussions.

\section{Funding}

Supported by grants from NIMH (R01MH101209) and Autism Speaks (\#2156) received by BZ.

\section{Availability of data and materials}

The datasets used and/or analyzed during the current study are available from the corresponding author on reasonable request.

\section{Authors' contributions}

Study design and conception: IMT, MAGC, BZ. Acquisition and analysis of data: IMT. Drafting of the manuscript: IMT, MAGC, BZ. All authors read and approved the final manuscript.

Ethics approval and consent to participate

The use of human post-mortem tissue for this study was approved by the Institutional Review Board (IRB) of Boston University. Experiments and procedures with animals were designed to minimize animal suffering and reduce the number of animals used. Detailed protocols of the procedures were approved by the Institutional Animal Care and Use Committee (IACUC) at Harvard Medical School and Boston University School of Medicine in

accordance with NIH guidelines (DHEW Publication no. [NIH] 80-22, revised 1996, Office of Science and Health Reports, DRR/NIH, Bethesda, Maryland, United States)

\section{Consent for publication}

Not applicable.

\section{Competing interests}

The authors declare that they have no competing interests.

\section{Publisher's Note}

Springer Nature remains neutral with regard to jurisdictional claims in published maps and institutional affiliations.

\section{Author details}

${ }^{1}$ Human Systems Neuroscience Laboratory, Boston University, 635 Commonwealth Ave., Room 401D, Boston, MA 02215, USA. ${ }^{2}$ Program in Neuroscience, Boston University, Boston, MA 02215, USA. ${ }^{3}$ Neural Systems Laboratory, Boston University, Boston, MA 02215, USA.

Received: 5 December 2018 Accepted: 23 February 2019

Published online: 13 March 2019

\section{References}

1. Adorjan I, Ahmed B, Feher V, Torso M, Krug K, Esiri M, Chance SA, Szele FG (2017) Calretinin interneuron density in the caudate nucleus is lower in autism spectrum disorder. Brain 140:2028-2040. https:/doi.org/10.1093/brain/awx131

2. Bachevalier J, Loveland KA (2006) The orbitofrontal-amygdala circuit and self-regulation of social-emotional behavior in autism. Neurosci Biobehav Rev 30:97-117

3. Bailey A, Luthert P, Dean A, Harding B, Janota I, Montgomery M, Rutter M, Lantos P (1998) A clinicopathological study of autism. Brain 121(Pt 5):889-905

4. Barbaria EM, Kohl B, Buhren BA, Hasenpusch-Theil K, Kruse F, Kury P, Martini R, Muller HW (2009) The alpha-chemokine CXCL14 is up-regulated in the sciatic nerve of a mouse model of Charcot-Marie-tooth disease type $1 \mathrm{~A}$ and alters myelin gene expression in cultured Schwann cells. Neurobiol Dis 33: 448-458. https://doi.org/10.1016/j.nbd.2008.11.014

5. Barbas H (1986) Pattern in the laminar origin of corticocortical connections. J Comp Neurol 252:415-422

6. Barbas H, Pandya DN (1989) Architecture and intrinsic connections of the prefrontal cortex in the rhesus monkey. J Comp Neurol 286:353-375

7. Barbas H, Rempel-Clower N (1997) Cortical structure predicts the pattern of corticocortical connections. Cereb Cortex 7:635-646

8. Bauman M, Kemper TL (1985) Histoanatomic observations of the brain in early infantile autism. Neurology 35:866-874

9. Bauman ML, Kemper TL (2003) The neuropathology of the autism spectrum disorders: what have we learned? NovartisFoundSymp 251:112-122

10. Berger B, Trottier S, Verney C, Gaspar P, Alvarez C (1988) Regional and laminar distribution of the dopamine and serotonin innervation in the macaque cerebral cortex: a radioautographic study. J Comp Neurol 273: 99-119

11. Boldog E, Bakken TE, Hodge RD, Novotny M, Aevermann BD, Baka J, Borde S, Close JL, Diez-Fuertes F, Ding SL et al (2018) Transcriptomic and morphophysiological evidence for a specialized human cortical GABAergic cell type. In: Nat Neurosci 21, pp 1185-1195. https://doi.org/ 10.1038/s41593-018-0205-2 
12. Buard I, Rogers SJ, Hepburn S, Kronberg E, Rojas DC (2013) Altered oscillation patterns and connectivity during picture naming in autism. Front Hum Neurosci 7:742. https://doi.org/10.3389/fnhum.2013.00742

13. Buxhoeveden DP, Semendeferi K, Buckwalter J, Schenker N, Switzer R, Courchesne $E$ (2006) Reduced minicolumns in the frontal cortex of patients with autism. Neuropathol Appl Neurobiol 32:483-491

14. Camacho J, Ejaz E, Ariza J, Noctor SC, Martinez-Cerdeno V (2014) RELNexpressing neuron density in layer I of the superior temporal lobe is similar in human brains with autism and in age-matched controls. Neurosci Lett 579:163-167. https://doi.org/10.1016/j.neulet.2014.07.031

15. Campbell MJ, Lewis DA, Foote SL, Morrison JH (1987) Distribution of choline acetyltransferase-, serotonin-, dopamine-B-hydroxylase-, tyrosine hydroxylase-immunoreactive fibers in monkey primary auditory cortex. J Comp Neurol 261:209-220

16. Casanova MF, Buxhoeveden DP, Switala AE, Roy E (2002) Minicolumnar pathology in autism. Neurology 58:428-432

17. Cauller L (1995) Layer I of primary sensory neocortex: where top-down converges upon bottom-up. Behavioral Brain Research 71:163-170

18. Cavanagh JF, Cohen MX, Allen JJ (2009) Prelude to and resolution of an error: EEG phase synchrony reveals cognitive control dynamics during action monitoring. J Neurosci 29:98-105. https://doi.org/10.1523/JNEUROSCl.4137-08.2009

19. Choi BH (1988) Developmental events during the early stages of cerebral cortical neurogenesis in man. A correlative light, electron microscopic, immunohistochemical and Golgi study. Acta Neuropathol 75:441-447

20. Clayton MS, Yeung N, Cohen Kadosh R (2015) The roles of cortical oscillations in sustained attention. Trends Cogn Sci 19:188-195. https://doi. org/10.1016/j.tics.2015.02.004

21. Colombo JA, Reisin HD (2004) Interlaminar astroglia of the cerebral cortex: a marker of the primate brain. Brain Res 1006:126-131

22. Constantinidis C, Procyk E (2004) The primate working memory networks. Cogn AffectBehavNeurosci 4:444-465

23. Courchesne E, Mouton PR, Calhoun ME, Semendeferi K, Ahrens-Barbeau C, Hallet MJ, Barnes CC, Pierce K (2011) Neuron number and size in prefronta cortex of children with autism. JAMA 306:2001-2010. https://doi.org/10. 1001/jama.2011.1638

24. Courchesne E, Pierce K (2005) Why the frontal cortex in autism might be talking only to itself: local over-connectivity but long-distance disconnection. Curr Opin Neurobiol 15:225-230

25. Crino PB (2013) Evolving neurobiology of tuberous sclerosis complex. Acta Neuropathol 125:317-332. https://doi.org/10.1007/s00401-013-1085-x

26. Cruikshank SJ, Ahmed OJ, Stevens TR, Patrick SL, Gonzalez AN, Elmaleh M, Connors BW (2012) Thalamic control of layer 1 circuits in prefrontal cortex. J Neurosci 32:17813-17823. https://doi.org/10.1523/JNEUROSCl.3231-12.2012

27. Deco G, Ponce-Alvarez A, Hagmann P, Romani GL, Mantini D, Corbetta M (2014) How local excitation-inhibition ratio impacts the whole brain dynamics. J Neurosci 34:7886-7898. https://doi.org/10.1523/JNEUROSCI.5068-13.2014

28. Defelipe J (1997) Types of neurons, synaptic connections and chemical characteristics of cells immunoreactive for calbindin-D28K, parvalbumin and calretinin in the neocortex. J Chem Neuroanat 14:1-19

29. del Rio MR, DeFelipe J (1997) Synaptic connections of calretininimmunoreactive neurons in the human neocortex. J Neurosci 17:5143-5154

30. Ding SL, Rockland KS, Zheng DS (2000) Parvalbumin immunoreactive CajalRetzius and non-Cajal-Retzius neurons in layer I of different cortical regions of human newborn. Anat Embryol (Berl) 201:407-417

31. Dinstein I, Pierce K, Eyler L, Solso S, Malach R, Behrmann M, Courchesne E (2011) Disrupted neural synchronization in toddlers with autism. Neuron 70: 1218-1225

32. Dombrowski SM, Hilgetag CC, Barbas H (2001) Quantitative architecture distinguishes prefrontal cortical systems in the rhesus monkey. Cereb Cortex 11:975-988

33. D'Souza RD, Burkhalter A (2017) A laminar organization for selective Corticocortical communication. Front Neuroanat 11:71. https://doi.org/10.3389/ fnana.2017.00071

34. Ebert DH, Greenberg ME (2013) Activity-dependent neuronal signalling and autism spectrum disorder. Nature 493:327-337. https://doi.org/10.1038/ nature 11860

35. Ecker C, Andrews D, Dell'Acqua F, Daly E, Murphy C, Catani M, Thiebaut de Schotten M, Baron-Cohen S, Lai MC, al LMV (2016) Relationship between cortical Gyrification, white matter connectivity, and autism Spectrum disorder. In: Cereb cortex 26, pp 3297-3309. https://doi.org/ 10.1093/cercor/bhw098
36. Fan J, Bernardi S, Dam NT, Anagnostou E, Gu X, Martin L, Park Y, Liu X, Kolevzon A, Soorya L et al (2012) Functional deficits of the attentional networks in autism. Brain and behavior 2:647-660. https://doi.org/10.1002/ brb3.90

37. Fatemi SH (2011) Reelin, a marker of stress resilience in depression and psychosis. Neuropsychopharmacology 36:2371-2372. https://doi.org/10. 1038/npp.2011.169

38. Folsom TD, Fatemi SH (2013) The involvement of Reelin in neurodevelopmental disorders. Neuropharmacology 68:122-135. https://doi. org/10.1016/..neuropharm.2012.08.015

39. Frotscher M (1997) Dual role of Cajal-Retzius cells and reelin in cortical development. Cell Tissue Res 290:315-322

40. Gabbott PL (2016) "Subpial Fan cell" - a class of Calretinin neuron in layer 1 of adult monkey prefrontal cortex. Front Neuroanat 10:28. https://doi.org/10. 3389/fnana.2016.00028

41. Gabbott PL, Bacon SJ (1996) Local circuit neurons in the medial prefrontal cortex (areas 24a,b,c, 25 and 32) in the monkey: I. Cell morphology and morphometrics. J Comp Neurol 364:567-608

42. Gabbott PLA, Somogyi P (1986) Quantitative distribution of GABAimmunoreactive neurons in the visual cortex (area 17) of the cat. Exp Brain Res 61:323-331

43. Gallyas F (1979) Silver staining of myelin by means of physical development Neurol Res 1:203-209

44. García-Cabezas MA, Barbas H (2014) Area 4 has layer IV in adult primates. Eur J Neurosci 39:1824-1834

45. García-Cabezas MA, Barbas H, Zikopoulos B (2018) Parallel development of chromatin patterns, neuron morphology, and connections: potential for disruption in autism. Front Neuroanat 12:70. https://doi.org/10.3389/fnana. 2018.00070

46. García-Cabezas MA, John YJ, Barbas H, Zikopoulos B (2016) Distinction of neurons, glia and endothelial cells in the cerebral cortex: an algorithm based on cytological features. Front Neuroanat 10:107. https://doi.org/10. 3389/fnana.2016.00107

47. Garcia-Cabezas MA, Joyce MP, John Y, Zikopoulos B, Barbas H (2017) Mirror trends of plasticity and stability indicators in primate prefrontal cortex. Eur J Neurosci 46:2392-2405

48. Ghashghaei HT, Hilgetag CC, Barbas H (2007) Sequence of information processing for emotions based on the anatomic dialogue between prefrontal cortex and amygdala. Neurolmage 34:905-923

49. Gibson JR, Bartley AF, Hays SA, Huber KM (2008) Imbalance of neocortical excitation and inhibition and altered UP states reflect network hyperexcitability in the mouse model of fragile X syndrome. J Neurophysiol 100:2615-2626

50. Gonzalez-Riano C, Tapia-Gonzalez S, Garcia A, Munoz A, DeFelipe J, Barbas C (2017) Metabolomics and neuroanatomical evaluation of post-mortem changes in the hippocampus. Brain Struct Funct 222:2831-2853. https://doi. org/10.1007/s00429-017-1375-5

51. Green SA, Hernandez L, Tottenham N, Krasileva K, Bookheimer SY, Dapretto M (2015) Neurobiology of sensory Overresponsivity in youth with autism Spectrum disorders. JAMA Psychiatry 72:778-786. https://doi.org/10.1001/ jamapsychiatry.2015.0737

52. Hashemi E, Ariza J, Rogers H, Noctor SC, Martinez-Cerdeno V (2016) The number of Parvalbumin-expressing interneurons is decreased in the medial prefrontal cortex in autism. Cereb Cortex. https://doi.org/10.1093/cercor/ bhw021

53. Hashimoto-Torii K, Torii M, Sarkisian MR, Bartley CM, Shen J, Radtke F, Gridley T, Sestan N, Rakic P (2008) Interaction between Reelin and notch signaling regulates neuronal migration in the cerebral cortex. Neuron 60:273-284

54. Hendry SHC, Schwark HD, Jones EG, Yan J (1987) Numbers and proportions of GABA-immunoreactive neurons in different areas of monkey cerebral cortex. J Neurosci 7:1503-1519

55. Hornung JP, de Tribolet N (1994) Distribution of GABA-containing neurons in human frontal cortex: a quantitative immunocytochemical study. AnatEmbryol(Berl) 189:139-145

56. Houser CR, Hendry SH, Jones EG, Vaughn JE (1983) Morphological diversity of immunocytochemically identified GABA neurons in the monkey sensorymotor cortex. J Neurocytol 12:617-638

57. Inan M, Zhao M, Manuszak M, Karakaya C, Rajadhyaksha AM, Pickel VM, Schwartz TH, Goldstein PA, Manfredi G (2016) Energy deficit in parvalbumin neurons leads to circuit dysfunction, impaired sensory gating and social disability. Neurobiol Dis 93:35-46. https://doi.org/10.1016/j.nbd.2016.04.004 
58. Innocenti GM (2011) Development and evolution: two determinants of cortical connectivity. Prog Brain Res 189:65-75. https://doi.org/10.1016/B9780-444-53884-0.00018-X

59. Jochaut D, Lehongre K, Saitovitch A, Devauchelle AD, Olasagasti I, Chabane N, Zilbovicius M, Giraud AL (2015) Atypical coordination of cortical oscillations in response to speech in autism. Front Hum Neurosci 9:171. https://doi.org/10.3389/fnhum.2015.00171

60. Jones EG (2007) The thalamus. Cambridge University Press, New York City

61. Just MA, Cherkassky VL, Keller TA, Minshew NJ (2004) Cortical activation and synchronization during sentence comprehension in high-functioning autism: evidence of underconnectivity. Brain 127:1811-1821

62. Kaes T (1907) Die Großhirnrinde des Menschen in ihren Maßen und in ihrem Fasergehalt - Ein gehirnanatomischer Atlas mit erläuterndem Text. Verlag von Gustav Fischer, City

63. Kana RK, Keller TA, Cherkassky VL, Minshew NJ, Just MA (2009) Atypica frontal-posterior synchronization of theory of mind regions in autism during mental state attribution. SocNeurosci 4:135-152

64. Kapfhammer JP, Schwab ME (1994) Inverse patterns of myelination and GAP-43 expression in the adult CNS: neurite growth inhibitors as regulators of neuronal plasticity? J Comp Neurol 340:194-206

65. Keehn B, Muller RA, Townsend J (2013) Atypical attentional networks and the emergence of autism. Neurosci Biobehav Rev 37:164-183. https://doi. org/10.1016/j.neubiorev.2012.11.014

66. Keehn B, Nair A, Lincoln AJ, Townsend J, Muller RA (2016) Under-reactive but easily distracted: an fMRI investigation of attentional capture in autism spectrum disorder. Developmental cognitive neuroscience 17:46-56. https:// doi.org/10.1016/j.den.2015.12.002

67. Khan S, Gramfort A, Shetty NR, Kitzbichler MG, Ganesan S, Moran JM, Lee SM, Gabrieli JD, Tager-Flusberg HB, Joseph RM et al (2013) Local and long-range functional connectivity is reduced in concert in autism spectrum disorders. Proc Natl Acad Sci USA 110:3107-3112. https://doi.org/10.1073/pnas.1214533110

68. Kitzbichler MG, Khan S, Ganesan S, Vangel MG, Herbert MR, Hamalainen MS, Kenet T (2014) Altered development and multifaceted band-specific abnormalities of resting state networks in autism. Biol Psychiatry. https://doi. org/10.1016/j.biopsych.2014.05.012

69. Kondo H, Osaka N, Osaka M (2004) Cooperation of the anterior cingulate cortex and dorsolateral prefrontal cortex for attention shifting. Neuroimage 23:670-679

70. Kuang XL, Zhao XM, Xu HF, Shi YY, Deng JB, Sun GT (2010) Spatio-temporal expression of a novel neuron-derived neurotrophic factor (NDNF) in mouse brains during development. BMC Neurosci 11:137. https://doi.org/10.1186/ 1471-2202-11-137

71. Kumar A, Sundaram SK, Sivaswamy L, Behen ME, Makki MI, Ager J, Janisse J, Chugani HT, Chugani DC (2009) Alterations in frontal lobe tracts and Corpus callosum in young children with autism Spectrum disorder. Cereb Cortex 20:2103-2113

72. Lakatosova S, Ostatnikova D (2012) Reelin and its complex involvement in brain development and function. Int J Biochem Cell Biol 44:1501-1504. https://doi.org/10.1016/j.biocel.2012.06.002

73. Larrain-Valenzuela J, Zamorano F, Soto-lcaza P, Carrasco X, Herrera C, Daiber F, Aboitiz F, Billeke P (2017) Theta and alpha oscillation impairments in autistic Spectrum disorder reflect working memory deficit. Sci Rep 7:14328. https://doi.org/10.1038/s41598-017-14744-8

74. Lebel C, Walker L, Leemans A, Phillips L, Beaulieu C (2008) Microstructural maturation of the human brain from childhood to adulthood. Neuroimage 40:1044-1055. https://doi.org/10.1016/j.neuroimage.2007.12.053

75. Lisman J, Yasuda R, Raghavachari S (2012) Mechanisms of CaMKII action in long-term potentiation. Nat Rev Neurosci 13:169-182. https://doi.org/10. 1038/nrn3192

76. Loveland KA, Bachevalier J, Pearson DA, Lane DM (2008) Fronto-limbic functioning in children and adolescents with and without autism. Neuropsychologia 46:49-62

77. Mai JK, Majtanik M, Paxinos G (2015) Atlas of the human brain. Academic Press - Elsevier, City

78. Marin-Padilla M (1990) 3-dimensional structural organization of layer-l of the human cerebral cortex - a golgi study. J Comp Neurol 299:89-105

79. Marin-Padilla M (1995) Prenatal development of fibrous (white matter) protoplasmic (gray matter), and layer I astrocytes in the human cerebral cortex: a Golgi study. J Comp Neurol 357:554-572

80. Marin-Padilla M (2011) Human motor cortex first Lamina and Grey matter special astrocytes: development and Cytoarchitecture. In: The human brain : prenatal development and structure. Springer, City, pp, pp 101-111
81. Marin-Padilla M (2011) Human motor cortex first Lamina: development and Cytoarchitecture. In: The human brain : prenatal development and structure. Springer, City, pp, pp 49-64

82. Marin-Padilla M (2015) Human cerebral cortex Cajal-Retzius neuron: development, structure and function. A Golgi study Front Neuroanat 9:21. https://doi.org/10.3389/fnana.2015.00021

83. Martinez-Cerdeno V, Galazo MJ, Cavada C, Clasca F (2002) Reelin immunoreactivity in the adult primate brain: intracellular localization in projecting and local circuit neurons of the cerebral cortex. hippocampus and subcortical regions Cereb Cortex 12:1298-1311

84. Medalla M, Barbas H (2006) Diversity of laminar connections linking periarcuate and lateral intraparietal areas depends on cortical structure. Eur Neurosci 23:161-179

85. Medalla M, Barbas H (2009) Synapses with inhibitory neurons differentiate anterior cingulate from dorsolateral prefrontal pathways associated with cognitive control. Neuron 61:609-620

86. Melchitzky DS, Eggan SM, Lewis DA (2005) Synaptic targets of calretinincontaining axon terminals in macaque monkey prefrontal cortex. Neuroscience 130:185-195

87. Mesulam MM, Hersh LB, Mash DC, Geula C (1992) Differential cholinergic innervation within functional subdivisions of the human cerebral cortex: a choline acetyltransferase study. J Comp Neurol 318:316-328

88. Meyer G, Gonzalez-Gomez M (2018) The heterogeneity of human CajalRetzius neurons. Semin Cell Dev Biol 76:101-111. https://doi.org/10.1016/j. semcdb.2017.08.059

89. Miller DJ, Duka T, Stimpson CD, Schapiro SJ, Baze WB, McArthur MJ, Fobbs AJ, Sousa AM, Sestan N, Wildman DE et al (2012) Prolonged myelination in human neocortical evolution. Proc Natl Acad Sci U S A 109:16480-16485. https://doi.org/10.1073/pnas.1117943109

90. Mrzljak L, Uylings HBM, Kostovic I, van Eden CG (1988) Prenatal development of neurons in the human prefrontal cortex: I. A qualitative golgi study. J Comp Neurol 271:355-386

91. Nácher V, Hassani SA, Womelsdorf T (2018) Asymmetric effective connectivity between primate anterior cingulate and lateral prefrontal cortex revealed by electrical microstimulation. Brain Struct Funct in press: https://doi.org/10.1007/s00429-018-1806-y

92. Nieuwenhuys R (2013) The myeloarchitectonic studies on the human cerebral cortex of the Vogt-Vogt school, and their significance for the interpretation of functional neuroimaging data. Brain Struct Funct 218:303352. https://doi.org/10.1007/s00429-012-0460-z

93. Nishikawa S, Goto S, Hamasaki T, Yamada K, Ushio Y (2002) Involvement of reelin and Cajal-Retzius cells in the developmental formation of vertical columnar structures in the cerebral cortex: evidence from the study of mouse presubicular cortex. Cereb Cortex 12:1024-1030

94. Oakley JC, Kalume F, Catterall WA (2011) Insights into pathophysiology and therapy from a mouse model of Dravet syndrome. Epilepsia 52(Suppl 2):5961. https://doi.org/10.1111/j.1528-1167.2011.03004.x

95. Pardo JV, Fox PT, Raichle ME (1991) Localization of a human system for sustained attention by positron emission tomography. Nature 349:61-64

96. Perge JA, Niven JE, Mugnaini E, Balasubramanian V, Sterling P (2012) Why do axons differ in caliber? J Neurosci 32:626-638. https://doi.org/10.1523/ JNEUROSCI.4254-11.2012

97. Peters A, Sethares C (2002) The effects of age on the cells in layer 1 of primate cerebral cortex. Cereb Cortex 12:27-36

98. Pizzarelli $R$, Cherubini $E$ (2011) Alterations of GABAergic signaling in autism spectrum disorders. Neural plasticity 2011:297153. https://doi.org/10.1155/ 2011/297153

99. Posner MI, Petersen SE (1990) The attention system of the human brain Annu Rev Neurosci 13:25-42

100. Rajkowska G, Goldman-Rakic PS (1995) Cytoarchitectonic definition of prefrontal areas in the normal human cortex: II. Variability in locations of areas 9 and 46 and relationship to the Talairach coordinate system. Cereb Cortex 5:323-337

101. Rajkowska G, Goldman-Rakic PS (1995) Cytoarchitectonic definition of prefrontal areas in the normal human cortex:I. Remapping of areas 9 and 46 using quantitative criteria. Cereb Cortex 5:307-322

102. Rakic S, Zecevic N (2003) Emerging complexity of layer I in human cerebral cortex. Cereb Cortex 13:1072-1083

103. Rubenstein JL, Merzenich MM (2003) Model of autism: increased ratio of excitation/inhibition in key neural systems. Genes Brain Behav 2:255-267

104. Ruzicka WB, Zhubi A, Veldic M, Grayson DR, Costa E, Guidotti A (2007) Selective epigenetic alteration of layer I GABAergic neurons isolated 
from prefrontal cortex of schizophrenia patients using laser-assisted microdissection. Mol Psychiatry 12:385-397. https://doi.org/10.1038/sj.mp. 4001954

105. Scemes E, Spray DC (2012) Astrocytes: wiring the brain. CRC, City

106. Schuman B, Machold R, Hashikawa Y, Fuzik J, Fishell G, Rudy B (2018) Four unique interneuron populations reside in neocortical layer 1.J Neurosci. https://doi.org/10.1523/JNEUROSCI.1613-18.2018

107. Schumann CM, Amaral DG (2006) Stereological analysis of amygdala neuron number in autism. J Neurosci 26:7674-7679

108. Schumann CM, Barnes CC, Lord C, Courchesne E (2009) Amygdala enlargement in toddlers with autism related to severity of social and communication impairments. Biol Psychiatry 66:942-949

109. Schumann CM, Hamstra J, Goodlin-Jones BL, Lotspeich L, Kwon H, Buonocore MH, Lammers CR, Reiss AL, Amaral DG (2004) The amygdala is enlarged in children but not adolescents with autism; the hippocampus is enlarged at all ages. J Neurosci 24:6392-6401

110. Shukla DK, Keehn B, Lincoln AJ, Muller RA (2010) White matter compromise of callosal and subcortical fiber tracts in children with autism spectrum disorder: a diffusion tensor imaging study. J Am Acad Child Adolesc Psychiatry 49:12691278, 1278 e1261-1262. https://doi.org/10.1016/j.jaac.2010.08.018

111. Shukla DK, Keehn B, Smylie DM, Muller RA (2011) Microstructural abnormalities of short-distance white matter tracts in autism spectrum disorder. Neuropsychologia 49:1378-1382

112. Smiley JF, Williams SM, Szigeti K, Goldman-Rakic PS (1992) Light and electron microscopic characterization of dopamine-immunoreactive axons in human cerebral cortex. J Comp Neurol 321:325-335. https://doi.org/10. 1002/cne.903210302

113. Solso S, Xu R, Proudfoot J, Hagler DJ Jr, Campbell K, Venkatraman V, Carter Barnes C, Ahrens-Barbeau C, Pierce K, Dale A et al (2016) Diffusion Tensor Imaging Provides Evidence of Possible Axonal Overconnectivity in Frontal Lobes in Autism Spectrum Disorder Toddlers. Biol Psychiatry 79:676-684. https://doi.org/10.1016/j.biopsych.2015.06.029

114. Spreafico R, Arcelli P, Frassoni C, Canetti P, Giaccone G, Rizzuti T, Mastrangelo M, Bentivoglio M (1999) Development of layer I of the human. cerebral cortex after midgestation: architectonic findings, immunocytochemical identification of neurons and glia, and in situ labeling of apoptotic cells. J Comp Neurol 410:126-142

115. Stoner R, Chow ML, Boyle MP, Sunkin SM, Mouton PR, Roy S, Wynshaw-Boris A, Colamarino SA, Lein ES, Courchesne E (2014) Patches of disorganization in the neocortex of children with autism. N Engl J Med 370:1209-1219. https://doi.org/10.1056/NEJMoa1307491

116. Sundaram SK, Kumar A, Makki MI, Behen ME, Chugani HT, Chugani DC (2008) Diffusion tensor imaging of frontal lobe in autism spectrum disorder. Cereb Cortex 18:2659-2665

117. Tamura R, Kitamura H, Endo T, Hasegawa N, Someya T (2010) Reduced thalamic volume observed across different subgroups of autism spectrum disorders. Psychiatry Res 184:186-188. https://doi.org/10.1016/j.pscychresns. 2010.07.001

118. Thatcher RW, North DM, Neubrander J, Biver CJ, Cutler S, Defina P (2009) Autism and EEG phase reset: deficient GABA mediated inhibition in thalamo-cortical circuits. Dev Neuropsychol 34:780-800. https://doi.org/10. 1080/87565640903265178

119. Tingjun C, Zhaohui L, Zhaocai J, Zihao L, Quangang X, Dehui H, Qing L, Shihui W (2015) Changes of CXCL12, CXCL14 and PDGF levels in the brain of patients with idiopathic demyelinating optic neuritis and neuromyelitis optica. $J$ Neuroimmunol 279:1-6. https://doi.org/10.1016/j.jneuroim.2014.12.004

120. Tsatsanis KD, Rourke BP, Klin A, Volkmar FR, Cicchetti D, Schultz RT (2003) Reduced thalamic volume in high-functioning individuals with autism. Biol Psychiatry 53:121-129

121. Verney C, Derer P (1995) Cajal-Retzius neurons in human cerebral cortex at midgestation show immunoreactivity for neurofilament and calciumbinding proteins. J Comp Neurol 359:144-153

122. von Economo C (1927/2009) Cellular structure of the human cerebral cortex (Translated and edited by Lazaros C. Triarhou). Karger, City

123. von Economo C, Koskinas GN (1925/2008) Atlas of cytoarchitectonics of the adult human cerebral cortex. Translated from the German original, revised and edited with an Introduction and additional appendix material by L. C. Triarhou. Karger, City

124. Wilson TW, Rojas DC, Reite ML, Teale PD, Rogers SJ (2007) Children and adolescents with autism exhibit reduced MEG steady-state gamma responses. Biol Psychiatry 62:192-197. https://doi.org/10.1016/j.biopsych.2006.07.002
125. y Ramón Cajal S (1904/2002) Textura del sistema nervioso del hombre y de los vertebrados. Tomo II, segunda parte. Gobierno de Aragón. Departamento de Cultura y Turismo, City

126. Zecevic N, Milosevic A, Rakic S, Marin-Padilla M (1999) Early development and composition of the human primordial plexiform layer: an immunohistochemical study. J Comp Neurol 412:241-254

127. Zecevic N, Rakic P (2001) Development of layer I neurons in the primate cerebral cortex. J Neurosci 21:5607-5619

128. Zikopoulos B, Barbas H (2007) Parallel driving and modulatory pathways link the prefrontal cortex and thalamus. PLoS One 2:e848. https://doi.org/10. 1371/journal.pone.0000848

129. Zikopoulos B, Barbas H (2010) Changes in prefrontal axons may disrupt the network in autism. J Neurosci 30:14595-14609

130. Zikopoulos B, Barbas H (2013) Altered neural connectivity in excitatory and inhibitory cortical circuits in autism. Front Hum Neurosci 7:609. https://doi. org/10.3389/fnhum.2013.00609

131. Zikopoulos B, Garcia-Cabezas MA, Barbas H (2018) Parallel trends in cortical grey and white matter architecture and connections in primates allow fine study of pathways in humans and reveal network disruptions in autism. PLoS Biol 16. https://doi.org/10.1371/journal.pbio.2004559

132. Zikopoulos B, John YJ, García-Cabezas MA, Bunce JG, Barbas H (2016) The intercalated nuclear complex of the primate amygdala. Neuroscience 330: 267-290. https://doi.org/10.1016/j.neuroscience.2016.05.052

133. Zikopoulos B, Liu X, Tepe J, Trutzer I, John YJ, Barbas H (2018) Opposite development of short- and long-range anterior cingulate pathways in autism. Acta Neuropathol. https://doi.org/10.1007/s00401-018-1904-1
Ready to submit your research? Choose BMC and benefit from:

- fast, convenient online submission

- thorough peer review by experienced researchers in your field

- rapid publication on acceptance

- support for research data, including large and complex data types

- gold Open Access which fosters wider collaboration and increased citations

- maximum visibility for your research: over $100 \mathrm{M}$ website views per year

At BMC, research is always in progress.

Learn more biomedcentral.com/submissions 